\title{
Theoretical Analysis for a Class of Rheonomous Affine Constraints on Configuration Manifolds-Part I: Fundamental Properties and Integrability/Nonintegrability Conditions
}

\author{
Tatsuya Kai \\ Department of Applied Electronics, Faculty of Industrial Science and Technology, Tokyo University of \\ Science, Chiba 278-8510, Japan \\ Correspondence should be addressed to Tatsuya Kai, kai@te.noda.tus.ac.jp \\ Received 6 May 2012; Accepted 4 July 2012 \\ Academic Editor: Wei-Chiang Hong \\ Copyright (C) 2012 Tatsuya Kai. This is an open access article distributed under the Creative \\ Commons Attribution License, which permits unrestricted use, distribution, and reproduction in \\ any medium, provided the original work is properly cited.

\begin{abstract}
We analyze a class of rheonomous affine constraints defined on configuration manifolds from the viewpoint of integrability/nonintegrability. First, we give the definition of $A$-rheonomous affine constraints and introduce, geometric representation their. Some fundamental properties of the $A$ rheonomous affine constrains are also derived. We next define the rheonomous bracket and derive some necessary and sufficient conditions on the respective three cases: complete integrability, partial integrability, and complete nonintegrability for the $A$-rheonomous affine constrains. Then, we apply the integrability/nonintegrability conditions to some physical examples in order to
\end{abstract} \\ confirm the effectiveness of our new results.
}

\section{Introduction}

In the research fields such as control theory and robotics, nonholonomic systems have been actively studied by a lot of researchers so far [1-5]. Nonholonomic systems are, in the simplest terms, defined as ones that are subject to nonintegrable constraints and whose behaviors must satisfy the constraints. That is to say, the word "nonholonomic" is equivalently used as "nonintegrable." We can easily find a lot of examples of nonholonomic systems: mobile cars $[6,7]$, trailers $[8,9]$, space robots $[10,11]$, acrobat robots $[12,13]$, a rolling ball or coin on a plain [1], underactuated manipulators [14-16], and so on. In past work on nonholonomic systems, linear constraints have been mainly treated. The linear constraints are represented as $B(q) \dot{q}=0, q \in \mathbf{R}^{n}, B(q) \in \mathbf{R}^{(n-m) \times n}$ and contain various examples such as mobile cars, a ball or a coin on a plain, and hopping robots $[14,15]$. Integrability and nonintegrability 
of the linear constraints can be determined by using the well-known Frobenius' theorem $[17,18]$, and they are strongly related to accessibility of nonlinear control systems that are derived from the linear constraints [4, 5, 7, 17-21]. Namely, researches on systems subject to the linear constraints have been highly developed by various approaches so far. Especially, researches on nonholonomic control systems can be mainly classified into the two research fields: kinematic systems and dynamic systems. Kinematic systems are directly derived from nonholonomic constraints, and in particular the linear constraints can be transformed into symmetrically affine control systems [7, 22, 23]. On the contrary, dynamic systems are derived from Euler-Lagrange equations with the constrained forces based on d'Alembert's principle $[21,24,25]$. There are two common characteristics for both kinematic and dynamic systems: (i) their linear approximated systems are uncontrollable and (ii) they are locally controllable, but not locally asymptotically stabilizable by any nonlinear smooth state feedback laws from Brockett's theorem $[4,26]$. Therefore, a lot of control methods such as time-variant feedback, discontinuous feedback, and switching control laws have been proposed to avoid Brockett's condition.

On the other hand, in nonholonomic systems, there exists another class of constraints called affine constraints. The affine constraints are represented as $A(q)+B(q) \dot{q}=0, q \in$ $\mathbf{R}^{n}, A(q) \in \mathbf{R}^{n-m}, B(q) \in \mathbf{R}^{(n-m) \times n}$ and contain some curious examples such as space robots with initial angular momenta (Figure 1(a)), a boat or a ship on a running river (Figure 1(b)), and a ball or a coin on a rotating table (Figures 1(c) and 1(d)). Obviously, the class of the affine constraints is larger than that of the linear constraints and covers a wide range of nonholonomic systems; however, theoretical analysis on the affine constraints had been hardly done. So, we focused on and researched the affine constraints from the viewpoints of both mathematics and control theory and derived various results: integrability/nonintegrability conditions of the affine constraints and foliation structures of configuration manifolds [27], integrating algorithms of integrable affine constraints [28], nonlinear control analysis and control of nonholonomic kinematic systems with affine constraints [29-32], modeling and theoretical analysis of nonholonomic dynamic systems with affine constraints [31,33], modeling and passivity analysis of nonholonomic Hamiltonian systems with affine constraints [34], generalized canonical transformation and passivity-based control of nonholonomic Hamiltonian systems with affine constraints [35], and near-optimal control of a 3D space robot with an initial angular momentum [36]. In a sequence of researches on the affine constraints, we obtained some important results: (a) complete nonintegrability (nonholonomicity) of the affine constraints is equivalent to strong accessibility of systems, (b) linear approximated systems of original systems with affine constraints are controllable under some conditions, and (c) some systems with affine constraints satisfy Brockett's condition and hence there is a possibility of local asymptotic stabilizability by nonlinear smooth state feedback laws. We can say that these characteristics are beyond the ones for the linear constraints and indicate a new control problem for nonholonomic control systems.

All the constraints that are dealt with in the researches above do not contain the time variable, that is, scleronomous constraints [1]. However, there exist nonholonomic mechanical systems whose constraints contain the time variable. For example, a boat or a ship on a running river with a time-varying stream (Figure 1(b)) and a coin or a ball on a rotating table at a time-varying angular velocity (Figures 1 (c) and $1(\mathrm{~d})$ ) are physical systems that are subject to affine constraints, which contain the time variable. These constraints are called rheonomous [1], especially, the class of A-rheonomous affine constraints that are represented as $A(t, q)+B(q) \dot{q}=0, q \in \mathbf{R}^{n}, A(t, q) \in \mathbf{R}^{n-m}, B(q) \in \mathbf{R}^{(n-m) \times n}$, that is, affine 


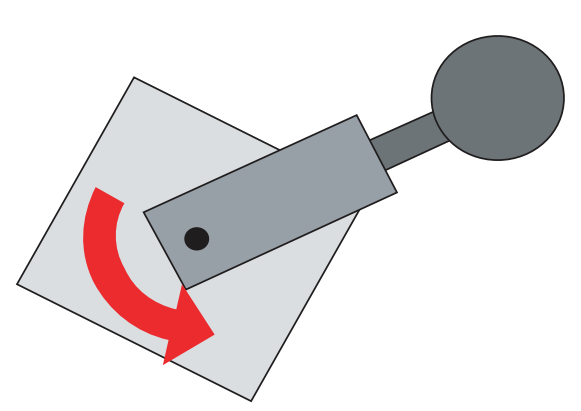

(a) Space Robot with Initial Angular Momentum

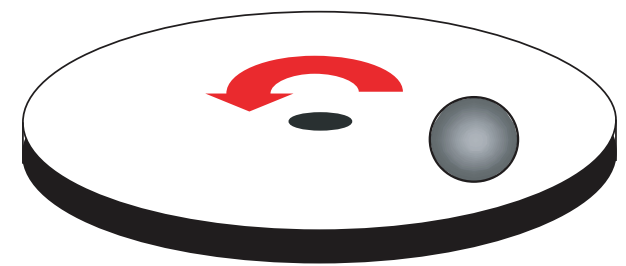

(c) Ball on Rotating Table

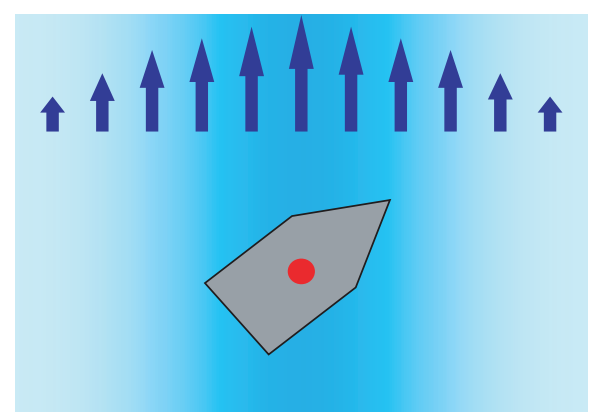

(b) Boat on Running River

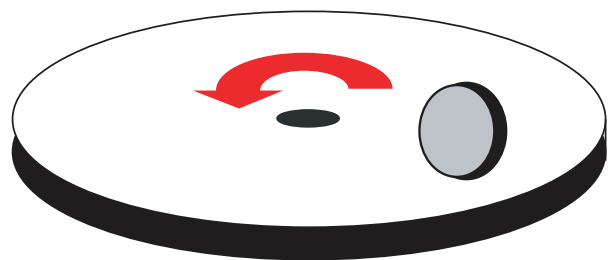

(d) Coin on Rotating Table

Figure 1: Examples of systems subject to affine constraints.

constraints whose term $A$ contains the time variable $t$ is important from an application standpoint. Moreover, the class of the $A$-rheonomous affine constraints is larger than that of the scleronomous affine constraints, and so we can say that the range of possible application extends more and more as shown in Figure 2. For analysis and control synthesis of mechanical systems subject to the rheonomous affine constraints, it is essential to consider them; however, the existing results on scleronomous affine constraints [27, 29] cannot be utilized. Hence, we need a new fundamental theory on the rheonomous affine constraints such as integrability/nonintegrability and foliation structures of configuration manifolds in order to apply control theory to such systems, and it is expected that the existence of the time variable in the constraints makes it more difficult to analyze them. In [37-41], rheonomous constraints have been dealt with in terms of Lagrangian mechanics, jet bundles, and almost-Poisson structures, but fundamental properties of rheonomous constraints such as integrability/nonintegrability and foliation structures of configuration manifolds have not been considered in these works.

This is the first paper of a series of papers on theoretical analysis of the $A$ rheonomous affine constraints defined on configuration manifolds. In this first paper, we aim at derivation of fundamental properties and integrability/nonintegrability conditions for the $A$-rheonomous affine constraints. specifically, integrability/nonintegrability analysis of the $A$-rheonomous affine constraints is one of the most basic and important concepts. On the other hand, the second paper [42] focuses on analysis of foliation structures of configuration manifolds with the $A$-rheonomous affine constraints and development of integrating algorithms for integrable $A$-rheonomous affine constraints.

This paper is organized as follows. In Section 2, we first give a definition of the Arheonomous affine constraints and explain their geometric representation. we also derive some fundamental properties of the $A$-rheonomous affine constraints. Next, Section 3 introduces the rheonomous bracket that is a new operator and plays an important role, and 


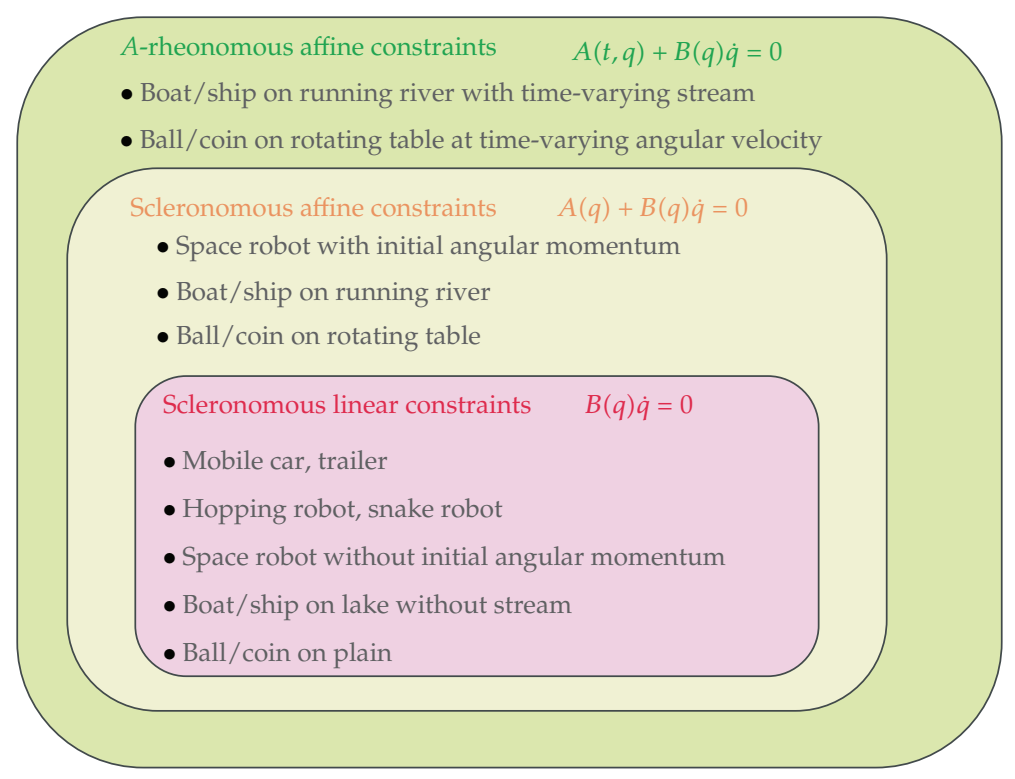

Figure 2: Classes of constraints and examples.

presents necessary and sufficient conditions for the $A$-rheonomous affine constraints in the three cases: complete integrability, partial integrability, and complete nonintegrability. Then, we apply our new results to some physical examples in order to confirm their effectiveness. Throughout this paper, manifolds, submanifolds, functions, vector fields, distributions, and differential forms are all assumed to be smooth.

\section{Rheonomous Affine Constraints}

\subsection{Definition}

In this subsection, we give a definition of rheonomous affine constraints. We denote the time variable by $t \in \mathbf{R}$ and a time interval by $I \subset \mathbf{R}$. Let $Q$ be an $n$-dimensional configuration manifold and $q=\left[q_{1} \cdots q_{n}\right]^{\top} \in \mathbf{R}^{n}$ to a local coordinate of $Q$. Associated with $q$, we refer $\dot{q}=\left[\dot{q}_{1} \cdots \dot{q}_{n}\right]^{\top} \in T_{q} Q$ as a tangent vector field.

A set of $n-m(m<n)$ differential equations:

$$
A_{i}(t, q)+B_{i 1}(t, q) \dot{q}_{1}+\cdots+B_{i n}(t, q) \dot{q}_{n}=0, \quad i=1, \ldots, n-m,
$$

is called rheonomous affine constraints because the coefficients $A_{i}, B_{i j}(i=1, \ldots, n-m, j=$ $1, \ldots, n)$ depend on the time variable $t$. We now rewrite (2.1) as

$$
A(t, q)+B(t, q) \dot{q}=0
$$

where a rheonomous affine term $A(t, q) \in \mathbf{R}^{n-m}$ is a vector-valued function whose $i$ th entry is $A_{i}(t, q)$ and $B(t, q)$ is a matrix-valued function whose $i j$ th entry is $B_{i j}(t, q)$. 
In this paper, we consider a subclass of the rheonomous affine constraints (2.2) in the form

$$
A(t, q)+B(q) \dot{q}=0
$$

that is to say, only the rheonomous affine term $A$ depends on the time variable $t$. We call (2.3) A-rheonomous affine constraints. It must be noted that this class of the $A$-rheonomous affine constraints contains some important examples of mechanical systems as mentioned in Section 1 . Now, we assume a sufficient condition on independency of the $A$-rheonomous affine constraints (2.3) as follows.

Assumption 2.1. The coefficient matrix $B(q)$ of the A-rheonomous affine constraints (2.3) has a row full-rank at any point $q \in Q$, that is,

$$
\operatorname{rank} B(q)=n-m, \quad \forall q \in Q,
$$

holds.

Then, we here give a definition on classification of points in the configuration manifold $Q$ in terms of the rheonomous affine term in the $A$-rheonomous affine constraints (2.3) as follows.

Definition 2.2. For the rheonomous affine term $A(t, q)$ of the $A$-rheonomous affine constraints (2.3) and a time interval $I \subset \mathbf{R}$, a point $q \in Q$ such that $A(t, q) \neq 0$, for all $t \in I$, holds is called a rheonomous affine regular point. On the other hand, a point $q \in Q$ such that $A(t, q)=$ 0 , for all $t \in I$, holds is called a rheonomous affine equilibrium point.

From Definition 2.2, we can see that each point in $Q$ is classified into either a rheonomous affine regular point or a rheonomous affine equilibrium point.

\subsection{Geometric Representation and Some Properties}

This subsection introduces a geometric representation method and provides some fundamental properties of the $A$-rheonomous affine constraints.

From (2.4) in Assumption 2.1, the $n-m$ row vectors of $B(q)$ in the $A$-rheonomous affine constraints (2.3) are independent of each other at any point $q \in Q$. Hence, we here consider $m$ vectors that are independent of each other and annihilators of the $n-m$ row vectors of $B(q)$ and denote them by $Y_{1}, \ldots, Y_{m}$ as vector fields on $Q$. In addition, we also denote a space spanned by $Y_{1}, \ldots, Y_{m}$, that is, a distribution on $Q$ by

$$
D:=\operatorname{span}\left\{Y_{1}, \ldots, Y_{m}\right\}
$$

Since the basial vectors of $D: Y_{1}, \ldots, Y_{m}$ are independent of each other at any point $q \in Q, D$ is a nonsingular distribution, that is,

$$
\operatorname{dim} D(q)=m, \quad \forall q \in Q
$$

holds. 


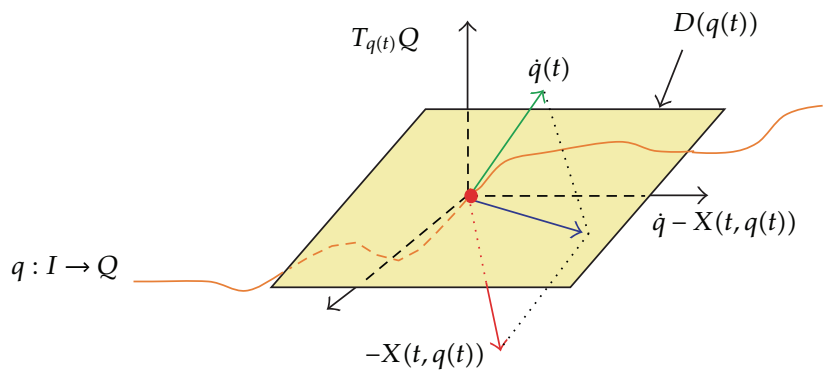

Figure 3: Geometric representation of $A$-rheonomous affine constraints.

In order to represent the $A$-rheonomous affine constraints geometrically, we now introduce an important vector field on $Q$. A curve $q: I \rightarrow Q$ is said to be satisfying the $A$ rheonomous affine constraints (2.3) if for a vector field on $Q: X$ and the generalized velocity of $q: \dot{q} \in T_{q(t)} Q$,

$$
\dot{q}(t)-X(t, q(t)) \in D(q(t)), \quad \forall t \in I,
$$

holds as shown in Figure 3. $X$ is called a rheonomous affine vector field. This definition is an extension of the one for the scleronomous affine constraints that do not contain the time variable [27].

Now, we show essential characteristics of the rheonomous affine vector field $X$ in the following proposition.

Proposition 2.3. For the A-rheonomous affine constraints (2.3), the component of the rheonomous affine vector field $X$, and a time interval $I \subset \mathbf{R}$,

$$
A(t, q)+B(q) X(t, q)=0, \quad \forall q \in Q, \forall t \in I,
$$

holds.

Proof. Assume that a velocity vector $\dot{q} \in T_{q} Q$ at a point $q \in Q$ satisfies the rheonomous affine constraints (2.3). Since $\dot{q}-X(t, q) \in D(q)$ holds, we obtain

$$
\dot{q}-X(t, q)=\alpha_{1}(t, q) Y_{1}(q)+\cdots+\alpha_{m}(t, q) Y_{m}(q)
$$

where $\alpha_{1}(t, q), \ldots, \alpha_{m}(t, q)$ are functions on $Q$. Now, multiply the left-hand side of (2.9) by $B(q)$. Since the row vectors of $B(q)$ are annihilators of $Y_{1}(q), \ldots, Y_{m}(q)$, we then have

$$
B(q)\{\dot{q}-X(t, q)\}=0
$$

Furthermore, using (2.3), we can rewrite (2.10) as

$$
B(q) \dot{q}-A(t, q)=0
$$

and hence we obtain (2.8). 
Consequently, under the preliminaries shown above, the A-rheonomous affine constraints (2.3) can be geometrically represented as the next definition.

Definition 2.4. The A-rheonomous affine constraints (2.3) are geometrically represented by a pair $(D, X)$, where $D$ is an $m$-dimensional distribution defined by (2.5) and $X$ is called $a$ rheonomous affine vector field and satisfies (2.8).

In order to derive some conditions on integrability/nonintegrability for $A$ rheonomous affine constraints (2.3), their geometric representation is quite important. For the geometric representation of the A-rheonomous affine constraints shown in Definition 2.4, the next proposition can be derived.

Proposition 2.5. For the geometric representation of the A-rheonomous affine constraints (2.3); $(D, X)$ and a time interval $I \subset \mathbf{R}$, a point $q \in Q$ is a rheonomous equilibrium point if and only if

$$
X(t, q(t)) \in D(q(t)), \quad \forall t \in I,
$$

holds. On the other hand, a point $q \in Q$ is a rheonomous regular point if and only if

$$
X(t, q(t)) \notin D(q(t)), \quad \forall t \in I,
$$

holds.

Proof. If $q \in Q$ is a rheonomous affine equilibrium point, that is, $A(q)=0$ holds, we have $B(q) X(q)=0$ and hence $X(t, q) \in D(q(t))$, for all $t \in I$, is derived from (2.3). Conversely, if $X(t, q) \in D(q(t))$ holds at a point $q \in Q$, that is, $B(q) X(q)=0$, then we have $A(q)=0$ from (2.3), and it turns out that the point is a rheonomous affine equilibrium point. Hence, a point $q \in Q$ is a rheonomous equilibrium point if and only if $X(t, q) \in D(q(t))$, for all $t \in I$, holds.

Next, all the points in $Q$ are classified into either rheonomous affine regular points or rheonomous affine equilibrium points from Definition 2.2. Therefore, a point $q \in Q$ is a rheonomous regular point if and only if $X(t, q) \notin D(q(t)), \forall t \in I$ holds. Consequently, we have completed the proof of this proposition.

\section{Integrability and Nonintegrability Conditions}

\subsection{Rheonomous Bracket}

This section presents some necessary and sufficient conditions for the A-rheonomous affine constraints (2.3) in the three cases of complete integrability, partial nonintegrability, and complete nonintegrability. First of all, we give a definition of a new operator called rheonomous bracket in this subsection. The normal Lie bracket for two vector fields $Z, W$ is defined as

$$
[Z, W]:=\frac{\partial W}{\partial q} Z-\frac{\partial Z}{\partial q} W
$$


Now, in order to derive some conditions for the $A$-rheonomous affine constraints, we define a new bracket, which can be interpreted as an extension of the normal Lie bracket above, as follows.

Definition 3.1. For the vector fields defined on $Q$ of the geometric representation of the $A$ rheonomous affine constraints $(2.2) ; X, Y_{1}, \ldots, Y_{m}$, the rheonomous bracket is an operator, $\langle\cdot, \cdot\rangle$ : $T Q \times T Q \rightarrow T Q$ that satisfies the next three properties.

(a) For a rheonomous affine vector field $X$,

$$
\langle X, X\rangle=0
$$

holds.

(b) $D_{0}$ is defined as a set of vector fields that consists of $Y_{1}, \ldots, Y_{m}$ and iterated rheonomous brackets of $X, Y_{1}, \ldots, Y_{m}$ and does not contain $X$. For a rheonomous affine vector field $X$ and a vector field $Z \in D_{0}$,

$$
\begin{aligned}
& \langle X, Z\rangle=\frac{\partial Z}{\partial t}+[X, Z], \quad Z \in D_{0} \\
& \langle Z, X\rangle=-\frac{\partial Z}{\partial t}+[Z, X], \quad Z \in D_{0}
\end{aligned}
$$

hold.

(c) For two vector fields $Z, W \in D_{0}$,

$$
\begin{gathered}
\langle Z, Z\rangle:=0, \quad Z \in D_{0} \\
\langle Z, W\rangle:=[Z, W], \quad Z, W \in D_{0}
\end{gathered}
$$

hold.

In Definition 3.1, it is the main characteristic that the rheonomous affine vector field $X$ is perceived as special, and this yields an additional term of a time differential of a vector field as shown in property (b). The rheonomous bracket will play important roles in not only derivation of integrability and nonintegrability conditions for the $A$-rheonomous affine constraints in the next subsections of this first paper but also analysis of foliation structure of configuration manifolds and development of integrating algorithms for the $A$-rheonomous affine constraints in the second paper [42]. It must be also noted that from Definition 3.1 the rheonomous bracket is equivalent to the normal Lie bracket for scleronomous affine constraints, that is, constraints that do not contain the time variable explicitly. In addition, it turns out from the next proposition that the rheonomous bracket has the important characteristics in common with the normal Lie bracket.

Proposition 3.2. For the vector fields on the geometric representation of the A-rheonomous affine constraints (2.2), $X, Y_{1}, \ldots, Y_{m}$, and the set of iterated vector fields of them, $D_{0}$, the following properties (a), (b), and (c) hold. 
(a) Bilinearlity:

$$
\begin{gathered}
\langle X, a Z+b W\rangle=a\langle X, Z\rangle+b\langle X, W\rangle, \\
\langle a Z+b W, X\rangle=a\langle Z, X\rangle+b\langle W, X\rangle, \quad Z, W \in D_{0} .
\end{gathered}
$$

(b) Skew-symmetry:

$$
\langle X, Z\rangle=-\langle Z, X\rangle, \quad Z, W \in D_{0}
$$

(c) Jacobi's identity:

$$
\langle\langle X, Z\rangle, W\rangle+\langle\langle Z, W\rangle, X\rangle+\langle\langle W, X\rangle, Z\rangle=0, \quad Z, W \in D_{0}
$$

Proof. Based on the definition of the rheonomous bracket, we can calculate

$$
\begin{aligned}
\langle X, a Z+b W\rangle & =\frac{\partial(a Z+b W)}{\partial t}+[X, a Z+b W] \\
& =a \frac{\partial Z}{\partial t}+a[X, Z]+b \frac{\partial W}{\partial t}+b[X, W] \\
& =a\langle X, Z\rangle+b\langle X, W\rangle \\
\langle a Z+b W, X\rangle & =-\frac{\partial(a Z+b W)}{\partial t}+[a Z+b W, X] \\
& =-a \frac{\partial Z}{\partial t}+a[Z, X]-b \frac{\partial W}{\partial t}+b[W, X] \\
& =a\langle Z, X\rangle+b\langle W, X\rangle .
\end{aligned}
$$

Hence, we complete the proof of (a). Next, a simple calculation can show that

$$
\begin{aligned}
\langle X, Z\rangle & =\frac{\partial Z}{\partial t}+[X, Z]=-\left(-\frac{\partial Z}{\partial t}+[Z, X]\right) \\
& =-\langle Z, X\rangle .
\end{aligned}
$$


Therefore, (b) holds. Finally, we will prove (c). Since we can calculate

$$
\begin{aligned}
\langle\langle X, Z\rangle, W\rangle & =\left\langle\frac{\partial Z}{\partial t}+[X, Z], W\right\rangle \\
& =\left[\frac{\partial Z}{\partial t}, W\right]+[[X, Z], W] \\
& =\frac{\partial W}{\partial q} \frac{\partial Z}{\partial t}+\frac{\partial^{2} Z}{\partial t \partial q} W+[[X, Z], W] \\
\langle\langle Z, W\rangle, X\rangle & =-\frac{\partial\langle Z, W\rangle}{\partial t}+[\langle Z, W\rangle, X] \\
& =-\frac{\partial[Z, W]}{\partial t}+[[Z, W], X] \\
& =-\frac{\partial}{\partial t}\left(\frac{\partial W}{\partial q} Z-\frac{\partial Z}{\partial q} W\right)+[[Z, W], X] \\
& =-\frac{\partial^{2} W}{\partial t \partial q} Z-\frac{\partial W}{\partial q} \frac{\partial Z}{\partial t}+\frac{\partial^{2} Z}{\partial t \partial q} W+\frac{\partial Z}{\partial q} \frac{\partial W}{\partial t}+[[Z, W], X], \\
\langle\langle W, X\rangle, Z\rangle & =[\langle W, X\rangle, Z] \\
& =\left[-\frac{\partial W}{\partial t}+[W, X], Z\right] \\
& =\left[-\frac{\partial W}{\partial t}, Z\right]+[[W, X], Z] \\
& =\frac{\partial Z}{\partial q} \frac{\partial W}{\partial t}+\frac{\partial^{2} W}{\partial t \partial q} Z+[[W, X], Z],
\end{aligned}
$$

we obtain

$$
\begin{aligned}
& \langle\langle X, Z\rangle, W\rangle+\langle\langle Z, W\rangle, X\rangle+\langle\langle W, X\rangle, Z\rangle \\
& =[[X, Z], W]+[[Z, W], X]+[[W, X], Z]=0,
\end{aligned}
$$

where we utilize Jacobi's identity for the normal Lie bracket. Consequently, the proof of (c) is completed.

The properties in Proposition 2.5 can reduce the effort to calculate iterated rheonomous brackets in checking complete nonholonomicity of the given $A$-rheonomous affine constraints.

\subsection{Complete Integrability Conditions}

In this subsection and the next two subsections, we will investigate the integrability/nonintegrability of the $A$-rheonomous affine constraints (2.3). First, in this subsection, we 
consider the case where all the $n-m$ A-rheonomous affine constraints (2.3) can be integrated, that is to say, the complete integrability case.

If the $A$-rheonomous affine constraints (2.3) are completely integrable, we can integrate them and obtain their independent first integrals that are represented by only the time variable $t$ and the configuration variable $q$ and do not contain any differentials of the configuration variable. Since we can reduce the dimension of a system subject to constraints by using independent first integrals of them and transform the system into another system with no constraints, it is quite important to examine if the constraints are integrable. We now define a smallest and involutive time-varying distribution $C_{0}(t, q)$ which contains $Y_{1}, \ldots, Y_{m}$ and iterated rheonomous brackets of them, and satisfies $\langle X, W\rangle \in C_{0}$, for all $W \in C_{0}$, that is to say, $C_{0}$ is spanned by all the rheonomous brackets of $X, Y_{1}, \ldots, Y_{m}$ with the exception of $X$. Then, some necessary and sufficient conditions on complete integrability for the $A$ rheonomous affine constraints (2.3) are given by the following theorem.

Theorem 3.3. For the A-rheonomous affine constraints (2.3) defined on an n-dimensional manifold $Q$ and a time interval $I \subset \mathbf{R}$, the following statements (a)-(c) are equivalent to each other. If they hold, the A-rheonomous affine constraints (2.3) are said to be completely integrable or completely holonomic.

(a) There exist $n-m$ independent first integrals of the A-rheonomous affine constraints (2.3): $h_{1}(t, q), \ldots, h_{n-m}(t, q)$ such that

$$
\begin{gathered}
\frac{\partial h_{i}(t, q)}{\partial t}+X(t, q) h_{i}(t, q)=0, \quad i=1, \ldots, n-m, \forall q \in Q, \forall t \in I, \\
Z(t, q) h_{i}(t, q)=0, \quad \forall Z \in D, i=1, \ldots, n-m, \quad \forall q \in Q, \forall t \in I, \\
d h_{1} \wedge \cdots \wedge d h_{n-m}(t, q) \neq 0, \quad \forall q \in Q, \quad \forall t \in I,
\end{gathered}
$$

hold, where $d$ is the exterior differential operator defined on $Q$.

(b) There exists an m-dimensional time-varying integral manifold $S^{m}\left(t, q^{0}\right)$ of the $A$ rheonomous affine constraints (2.3) determined by an initial point $q^{0} \in Q$ such that

$$
T_{q} S^{m}\left(t, q^{0}\right)=D(t, q), \quad \forall q \in S^{m}\left(t, q^{0}\right), \forall t \in I,
$$

holds.

(c) For a smallest and involutive time-varying distribution $C_{0}$,

$$
\operatorname{dim} C_{0}(t, q)=m, \quad \forall q \in Q, \forall t \in I,
$$

holds.

Proof. First of all, the setting for the proof is given. We denote the product manifold of the space of the time variable $\mathbf{R}$ and the configuration manifold $Q$ by $\bar{Q}:=\mathbf{R} \times Q$ with the coordinate $\bar{q}:=\left[\begin{array}{ll}t & q^{\top}\end{array}\right]^{\top}$, and $\bar{Q}$ is called an expanded configuration manifold with the dimension 


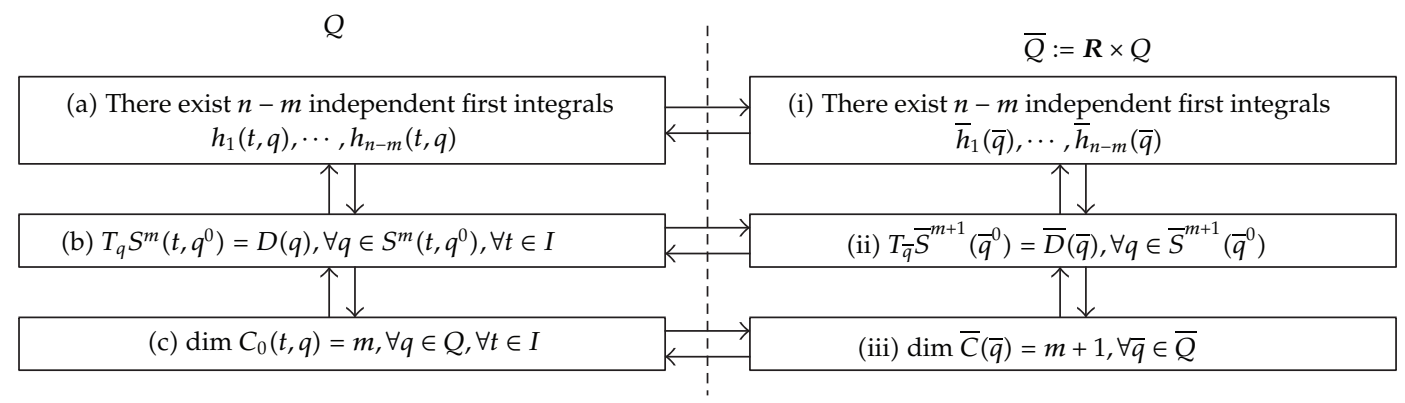

Figure 4: A diagram for the proof of Theorem 3.3.

$n+1$. Hence, the $A$-rheonomous affine constraints (2.3) defined on $Q$ can be represented by an expanded Pfaffian equation on $\bar{Q}$ :

$$
\bar{\Omega}(\bar{q})=0
$$

with $n-m$ expanded differential forms:

$$
\bar{\Omega}(\bar{q}):=A(t, q) d t+B(q) d q .
$$

Since the rheonomous affine vector field $X$ satisfies $A(t, q)+B(q) X(t, q)=0$ and the $m$ vector fields $Y_{1}, \ldots, Y_{m}$ satisfy $B(q) Y_{1}=\cdots=B(q) Y_{m}=0, m+1$ annihilators of the $n-m$ expanded differential forms (3.18) can be derived as

$$
\bar{X}=\frac{\partial}{\partial t} \oplus X, \quad \bar{Y}_{i}=0 \oplus Y_{i}, \quad i=1, \ldots, m
$$

We consider an $(m+1)$-dimensional expanded distribution on $\bar{Q}$ that is spanned by the $m+1$ vector fields $\bar{X}, \bar{Y}_{1}, \ldots, \bar{Y}_{m}$ and denote it by

$$
\bar{D}:=\operatorname{span}\left\{\bar{X}, \bar{Y}_{1}, \ldots, \bar{Y}_{m}\right\}
$$

We will prove this theorem in line with the diagram shown in Figure 4. Since it is possible to show that the statements (i), (ii), and (iii) in Figure 4 are equivalent to each other on $\bar{Q}$ by Frobenius' theorem $[17,19]$, all we have to do is prove the three equivalences between (a) and (i), (b) and (ii), and (c) and (iii).

We begin with the proof of the equivalence between (a) and (i). First, we prove (a) $\Rightarrow$ (i). Assume that (a) holds, that is, there exist $n-m$ independent first integrals $h_{1}(t, q), \ldots, h_{n-m}(t, q)$ of the $A$-rheonomous affine constraints (2.3) on $Q$ and they satisfy 
(3.12)-(3.14). Now, we define $\bar{h}_{1}(\bar{q}):=h_{1}(t, q), \ldots, \bar{h}_{n-m}(\bar{q}):=h_{n-m}(t, q)$ and show that these are $n-m$ independent first integrals of the expanded Pfaffian equation (3.17) on $\bar{Q}$, that is,

$$
\begin{gathered}
\bar{Z} \bar{h}_{j}=0, \quad \forall \bar{Z} \in \bar{D}, j=1, \ldots, n-m, \\
\overline{d h}_{1} \wedge \cdots \wedge \overline{d h}_{n-m} \neq 0
\end{gathered}
$$

hold, where $\bar{d}$ is the expanded exterior derivative operator defined on $\bar{Q}$. From the form of $\bar{X}$ and (3.12), we have

$$
\begin{aligned}
\bar{X} \bar{h}_{j} & =\frac{\partial \bar{h}_{j}}{\partial t}+\bar{X}_{1} \frac{\partial \bar{h}_{j}}{\partial q_{1}}+\cdots+\bar{X}_{n} \frac{\partial \bar{h}_{j}}{\partial q_{n}} \\
& =\frac{\partial h_{j}}{\partial t}+X_{1} \frac{\partial h_{j}}{\partial q_{1}}+\cdots+X_{n} \frac{\partial h_{j}}{\partial q_{n}} \\
& =\frac{\partial h_{j}}{\partial t}+X h_{j} \\
& =0, \quad j=1, \ldots, n-m .
\end{aligned}
$$

From the form of $Y_{i}$ and (3.13), we also obtain

$$
\begin{aligned}
\bar{Y}_{i} \bar{h}_{j} & =0 \cdot \frac{\partial}{\partial t}+\bar{Y}_{1} \frac{\partial \bar{h}_{j}}{\partial t}+\frac{\partial \bar{h}_{j}}{\partial q_{1}}+\cdots+\bar{Y}_{n} \frac{\partial \bar{h}_{j}}{\partial q_{n}} \\
& =Y_{1} \frac{\partial h_{j}}{\partial q_{1}}+\cdots+Y_{n} \frac{\partial h_{j}}{\partial q_{n}} \\
& =Y_{i} h_{j} \\
& =0, \quad i=1, \ldots, m, j=1, \ldots, n-m .
\end{aligned}
$$

Therefore, it turns out that (3.21) holds. Furthermore, calculating the left-hand side of (3.22), we have

$$
\begin{aligned}
\overline{d h}_{1} \wedge \cdots \wedge \overline{d h}_{n-m}= & \left(\frac{\partial h_{1}}{\partial t} d t+d h_{1}\right) \wedge \cdots \wedge\left(\frac{\partial h_{n-m}}{\partial t} d t+d h_{n-m}\right) \\
= & d h_{1} \wedge \cdots \wedge d h_{n-m}+\frac{\partial h_{1}}{\partial t} d t \wedge d h_{2} \wedge \cdots \wedge d h_{n-m} \\
& +\cdots+\frac{\partial h_{n-m}}{\partial t} d t \wedge d h_{1} \wedge \cdots \wedge d h_{n-m-1}
\end{aligned}
$$

and hence we can see that (3.22) holds under (3.14). So, the proof of (a) $\Rightarrow$ (i) is completed. Conversely, we then prove $(\mathrm{a}) \Leftarrow(\mathrm{i})$. It is assumed that there exist $n-m$ independent first integrals of the expanded Pfaffian equation (3.17) on $\bar{Q}, \bar{h}_{1}(\bar{q}), \ldots, \bar{h}_{n-m}(\bar{q})$, and they satisfy (3.21) and (3.22). We define $h_{1}(t, q):=\bar{h}_{1}(\bar{q}), \ldots, h_{n-m}(t, q):=\bar{h}_{n-m}(\bar{q})$ and show that they are 
$n-m$ independent first integrals of the $A$-rheonomous affine constraints (2.3) on $Q$, that is to say, (3.12) and (3.13) hold. By using (3.21), we obtain

$$
\begin{gathered}
\frac{\partial}{\partial t}+X h_{j}=1 \cdot \frac{\partial}{\partial t}+X h_{j}=\bar{X} \bar{h}_{j}=0, \quad j=1, \ldots, n-m, \\
Y_{i} h_{j}=0+Y_{i} h_{j}=\bar{Y}_{i} \bar{h}_{j}=0, \quad i=1, \ldots, m, j=1, \ldots, n-m,
\end{gathered}
$$

and hence (3.12) holds. We now assume that $d h_{1} \wedge \cdots \wedge d h_{n-m}=0$, that is, $h_{1}, \ldots, h_{n-m}$ are not independent of each other. Then, without loss of generality, we can write

$$
h_{1}(t, q)=\sum_{i=2}^{n-m} a_{i} h_{i}(t, q)
$$

that is to say, $h_{1}$ is represented as a linear combination of $h_{2}, \ldots, h_{n-m}$ with coefficients $a_{i} \quad(i=$ $2, \ldots, n-m)$. We then have

$$
\begin{aligned}
\bar{h}_{1}(\bar{q}) & =\sum_{i=2}^{n-m} a_{i} \bar{h}_{i}(\bar{q}) \\
\overline{d h}_{1}(\bar{q}) & =\sum_{i=2}^{n-m} a_{i} \overline{d h}_{i}(\bar{q})
\end{aligned}
$$

Therefore, we can find that

$$
\overline{d h}_{1} \wedge \cdots \wedge \overline{d h}_{n-m}=\left(\sum_{i=2}^{n-m} a_{i} \overline{d h}_{i}\right) \wedge \overline{d h}_{2} \wedge \cdots \wedge \overline{d h}_{n-m}=0
$$

holds, and hence (3.29) is inconsistent with assumption (3.22). So, it can be confirmed that $d h_{1} \wedge \cdots \wedge d h_{n-m} \neq 0$ holds and $h_{1}(t, q), \ldots, h_{n-m}(t, q)$ are independent first integrals. Consequently, we have proven $(\mathrm{a}) \Leftarrow(\mathrm{i})$, and hence the proof of (a) $\Leftrightarrow(\mathrm{i})$ is finished.

Next, we prove the equivalence between (b) and (ii). We first prove (b) $\Leftarrow$ (ii). See also the illustration shown in Figure 5 for understanding the proof. Assume that for an $(m+1)$ dimensional submanifold in $\bar{Q}$ with the initial point $\bar{q}^{0} \in \bar{Q}: \bar{S}^{m+1}\left(\bar{q}^{0}\right)$,

$$
T_{\bar{q}} \bar{S}^{m+1}\left(\bar{q}^{0}\right)=\bar{D}(\bar{q}), \quad \bar{q} \in \bar{S}^{m+1}\left(\bar{q}^{0}\right)
$$

holds. Now, we denote a diffeomorphism that is a projection from $\bar{Q}$ to $Q$ at the time $t$ by $\pi_{t}:(t, q) \mapsto q(t)$ and its derivation by $d \pi_{t}: T_{\bar{q}(t)} \bar{Q} \rightarrow T_{q} Q$. Consider a decomposition of $D$ as $\bar{D}=: \bar{H} \oplus \bar{V}$, where $\bar{H}:=\operatorname{span}\left\{\bar{Y}_{1}, \ldots, \bar{Y}_{m}\right\}$. In addition, we set $\pi_{t_{0}}\left(\bar{q}^{0}\right)=: q^{0}, \mathrm{C} \pi_{t}(\bar{q})=: q$ and the projection of $\bar{S}^{m+1}\left(\bar{q}^{0}\right)$ on $\bar{Q}$ to $Q$ by $\pi_{t}$ is defined as $\pi_{t}\left(\bar{S}^{m+1}\left(\bar{q}^{0}\right)\right)=: S^{m}\left(t, q^{0}\right)$. Then, we can find that

$$
d \pi_{t}\left(T_{\bar{q}} \bar{S}^{m+1}\left(\bar{q}^{0}\right)\right)=d \pi_{t}\left(T_{q} S^{m}(t, q)\right)
$$




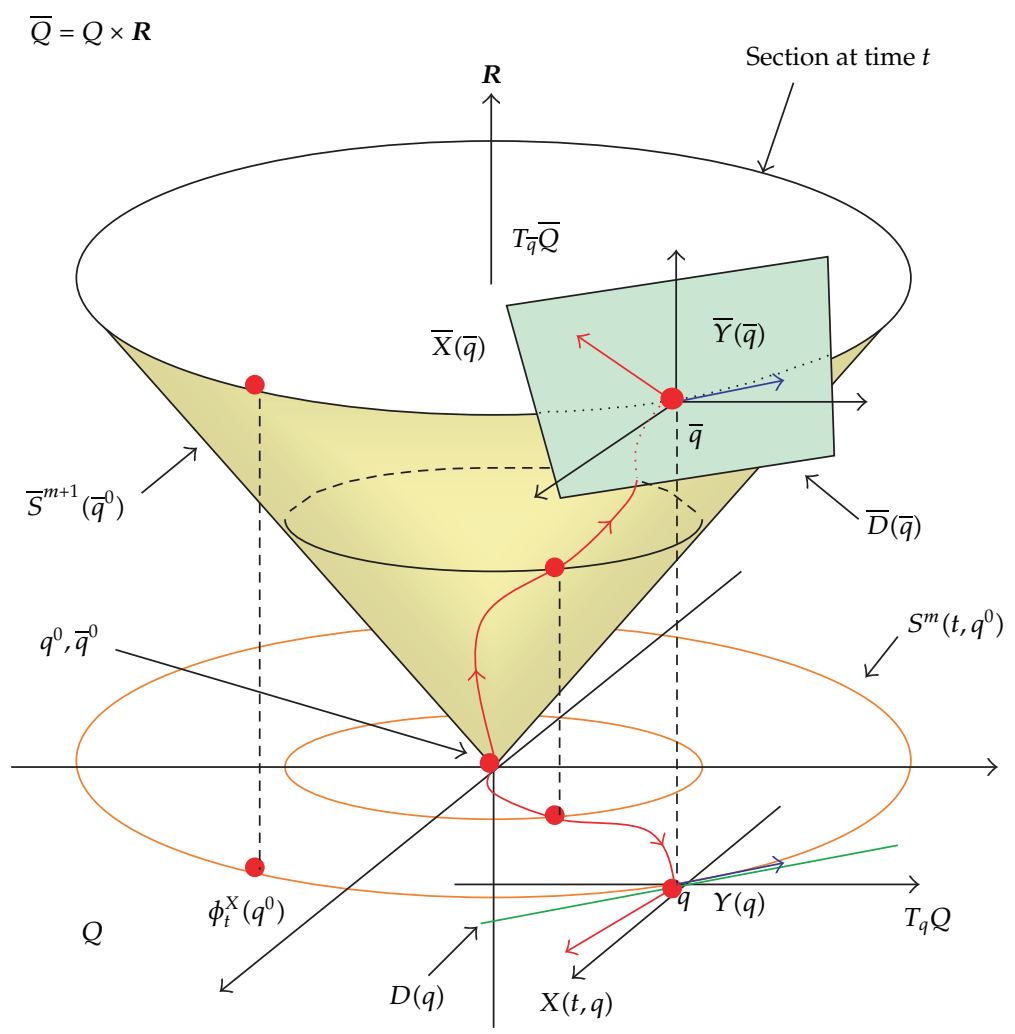

Figure 5: An illustration of $Q$ and $\bar{Q}(n=2, m=1)$.

holds, and hence from (3.19) and

$$
d \pi_{t}\left(\bar{Y}_{1}(\bar{q})\right)=Y_{1}(q), \ldots, d \pi_{t}\left(\bar{Y}_{m}(\bar{q})\right)=Y_{m}(q)
$$

we derive

$$
d \pi_{t}(\bar{D}(q))=d \pi_{t}(\bar{H}(q))=D(q)
$$

Therefore, from (3.30), (3.15) holds and we conclude $(\mathrm{b}) \Leftarrow$ (ii). In contrast, we can prove (b) $\Rightarrow$ (ii) by using the inverse mapping of $\pi_{t}: \pi_{t}^{-1}: q(t) \mapsto(t, q)$ in the same manner. Especially, from $S^{m}\left(t, q^{0}\right)$ on $Q$, we can construct an $(m+1)$-dimensional submanifold on $\bar{Q}$ as

$$
\bar{S}^{m+1}\left(\bar{q}^{0}\right):=\bigcup_{t \geq 0} \pi_{t}^{-1}\left(S^{m}\left(t, q^{0}\right)\right)
$$

So, the proof of (b) $\Rightarrow$ (ii) is completed, and hence we conclude that (b) $\Leftrightarrow$ (ii) holds.

Finally, we give a proof on the equivalence between (c) and (iii). We here define an involutive distribution $\bar{C}$ defined on $\bar{Q}$, which contains $\bar{X}, \bar{Y}_{1}, \ldots, \bar{Y}_{m}$ and iterated Lie brackets 
that consist of $\bar{X}, \bar{Y}_{1}, \ldots, \bar{Y}_{m}$. Therefore, a necessary and sufficient condition of the complete integrability for the expanded Pfaffian equation is given by

$$
\operatorname{dim} \bar{C}(t, q)=m+1, \quad \forall t \in I, \forall q \in Q
$$

(cf. Frobenius' theorem [17, 19]). Calculating the iterated Lie brackets that consist of $\bar{X}, \bar{Y}_{1}, \ldots, \bar{Y}_{m}$, we have

$$
\begin{aligned}
& {\left[\bar{X}(t, q), \bar{Y}_{i}(q)\right]=0 \oplus\left\langle X(t, q), Y_{i}(q)\right\rangle,} \\
& {\left[\bar{X}(t, q), \bar{X}(t, q), \bar{Y}_{i}(q)\right]=0 \oplus\left\langle X(t, q),\left\langle X(t, q), Y_{i}(q)\right\rangle\right\rangle, \ldots} \\
& {\left[\bar{Y}_{j}(q), \bar{Y}_{i}(q)\right]=0 \oplus\left\langle Y_{j}(q), Y_{i}(q)\right\rangle,} \\
& {\left[\bar{Y}_{k}(q),\left[\bar{Y}_{j}(q), \bar{Y}_{i}(q)\right]\right]=0 \oplus\left\langle Y_{k}(q),\left\langle Y_{j}(q), Y_{i}(q)\right\rangle\right\rangle, \ldots}
\end{aligned}
$$

We can see that $\bar{X}$ is independent of $\bar{Y}_{i}, \ldots, \bar{Y}_{m}$ and the iterated Lie brackets (3.36). Then, the necessary and sufficient condition (3.35) is changed into the condition such that $\bar{Y}_{1}, \ldots, \bar{Y}_{m}$ and the iterated Lie brackets which consist of $\bar{X}, \bar{Y}_{1}, \ldots, \bar{Y}_{m}$ span an $m$-dimensional space. From (3.19) and (3.36), we can consider only $Y_{1}, \ldots, Y_{m}$ on $Q$ instead of $\bar{Y}_{1}, \ldots, \bar{Y}_{m}$ on $\bar{Q}$ and iterated rheonomous brackets that consist of $X, Y_{1}, \ldots, Y_{m}$ on $Q$ instead of Lie brackets that consist of $\bar{X}, \bar{Y}_{1}, \ldots, \bar{Y}_{m}$ on $\bar{Q}$. Therefore, a necessary and sufficient condition of complete integrability for the rheonomous affine constraints (2.2) is that $Y_{1}, \ldots, Y_{m}$ and the iterated rheonomous brackets which consist of $X, Y_{1}, \ldots, Y_{m}$ span an $m$-dimensional space, that is, (3.16) holds. Consequently, we complete the proof of (c) $\Leftrightarrow$ (iii).

Since we have proved the equivalence between (a) and (i), (b) and (ii), and (c) and (iii), we have completed the proof of this theorem of the complete integrability of the $A$ rheonomous affine constraints (2.3).

It must be noted that though the A-rheonomous affine constraints (2.3) contain the time variable, conditions (a)-(c) in Theorem 3.3 are similar to the ones for the scleronomous affine constraints case $[27,29]$, and the rheonomous bracket plays an essential role. In order to the check complete integrability of the given $A$-rheonomous affine constraints, the condition (c) in Theorem 3.3 is very useful. By calculating some iterated rheonomous brackets of vector fields of their geometric representation and investigating the dimension of $C_{0}$, we can easily determine their complete integrability. Now, we consider a simple example in order to verify Theorem 3.3 as follows.

Example 3.4. We consider a 3-dimensional configuration manifold

$$
Q=\left\{q=\left[\begin{array}{l}
q_{1} \\
q_{2} \\
q_{3}
\end{array}\right] \in \mathbf{R}^{3} \mid q_{2}>0, q_{3}>0\right\}
$$


with $n=3$ and $A$-rheonomous affine constraints on $Q$

$$
\left.\begin{array}{c}
t q_{1} q_{2} q_{3} \\
0 \\
\{\} \\
A(t, q)
\end{array}\right]+\left[\begin{array}{ccc}
q_{2} q_{3} & q_{1} q_{3} & q_{1} q_{2} \\
0 & 1 & 0
\end{array}\right]\left[\begin{array}{l}
\dot{q}_{1} \\
\dot{q}_{2} \\
\dot{q}_{3}
\end{array}\right]=0
$$

with $m=1$. In addition, we consider a time interval $I:=\mathbf{R}$. We can see that Assumption 2.1 holds for (3.38). One of the geometric representations of (3.38) can be obtained as follows:

$$
X(t, q)=\left[\begin{array}{c}
-t q_{1} \\
0 \\
0
\end{array}\right], \quad Y(q)=\left[\begin{array}{c}
-q_{1} \\
0 \\
q_{3}
\end{array}\right] .
$$

Calculating a iterated rheonomous brackets for $X$ and $Y$ above, we obtain

$$
\langle X, Y\rangle=\frac{\partial Y}{\partial t}+[X, Y]=0+\frac{\partial Y}{\partial q} X-\frac{\partial X}{\partial q} Y=\left[\begin{array}{c}
t q_{1}-t q_{1} \\
0 \\
0
\end{array}\right]=0
$$

Hence, it turns out that all the iterated rheonomous brackets of $X$ and $Y$ are 0 . Therefore, we have

$$
C_{0}=\operatorname{span}\{Y\}
$$

and it is confirmed that

$$
\operatorname{dim} C_{0}=1, \quad \forall q \in Q, \forall t \in I,
$$

holds. From Theorem 3.3, we can see that the A-rheonomous affine constraints (3.38) are completely integrable. In fact, there exist two independent first integrals of (3.38):

$$
h_{1}(t, q)=q_{2}-q_{2}^{0}, \quad h_{2}(t, q)=\frac{q_{1} q_{3} e^{(1 / 2) t^{2}}}{q_{3}^{0}}-q_{1}^{0},
$$

where $q^{0}=\left[q_{1}^{0} q_{2}^{0} q_{3}^{0}\right]^{\top} \in Q$ is an initial point at the initial time. We can easily confirm that (3.43) satisfies (3.12)-(3.14) of condition (a) in Theorem 3.3, and hence (3.43) are independent first integrals of (3.38). In the second paper [42], we will derive an integrating algorithm for completely integrable $A$-rheonomous affine constraints and show how to calculate (3.43).

\subsection{Partial Integrability Conditions}

In this subsection, we next consider the case where some of the $A$-rheonomous affine constraints in (2.3) are integrable and the others are nonintegrable, that is to say, integrable 
constraints and nonintegrable ones are mixed. We call such a case partial integrability. Now, let us derive some necessary and sufficient conditions on the partial integrability of the $A$ rheonomous affine constraints (2.3) in the following theorem.

Theorem 3.5. For the A-rheonomous affine constraints (2.3) defined on an n-dimensional manifold $Q$ and a time interval $I \subset \mathbf{R}$, the following statements (a)-(c) are equivalent to each other. If they hold, the A-rheonomous affine constraints (2.3) are said to be kth order partially integrable or kth order partially holonomic, where $m<k<n$.

(a) There exist $n-k$ independent first integrals of the A-rheonomous affine constraints (2.3): $h_{1}(t, q), \ldots, h_{n-k}(t, q)$ such that

$$
\begin{gathered}
\frac{\partial h_{i}(t, q)}{\partial t}+X(t, q) h_{i}(t, q)=0, \quad i=1, \ldots, n-k, \forall q \in Q, \forall t \in I, \\
Z(t, q) h_{i}(t, q)=0, \quad \forall Z \in C_{0}, i=1, \ldots, n-k, \forall q \in Q, \forall t \in I, \\
d h_{1} \wedge \cdots \wedge d h_{n-k}(t, q) \neq 0, \quad \forall q \in Q, \forall t \in I,
\end{gathered}
$$

hold.

(b) There exists a $k$-dimensional time-varying integral manifold $S^{k}\left(t, q^{0}\right)$ of the A-rheonomous affine constraints (2.3) determined by an initial point $q^{0} \in Q$ such that

$$
T_{q} S^{k}\left(t, q^{0}\right)=C_{0}(t, q), \quad \forall q \in S^{k}\left(t, q^{0}\right), \forall t \in I,
$$

holds.

(c) For a smallest and involutive time-varying distribution $C_{0}$,

$$
\operatorname{dim} C_{0}(t, q)=k, \quad \forall q \in Q, \forall t \in I,
$$

(d) holds.

Proof. We prove this theorem along the diagram shown in Figure 6. Since similar to the proof of Theorem 3.3, we can show that the statements (i)-(iii) in Figure 6 are equivalent to each other by using Frobenius' theorem [17, 19], we will prove the three equivalences between (a) and (i), (b) and (ii), and (c) and (iii) to complete the proof.

First, we show the equivalence between (a) and (i). Define a new smallest and involutive distribution on $\bar{Q}, \bar{C}_{0}$, that contains all the iterated Lie brackets of $\bar{Y}_{1}, \ldots, \bar{Y}_{m}$ and iterated Lie brackets their and satisfies $[\bar{X}, \bar{Z}] \in \bar{C}_{0}$ for $\forall \bar{Z} \in \bar{C}_{0}$. That is to say, $\bar{C}_{0}$ is a subbundle in $T \bar{Q}$ that is spanned by $\bar{Y}_{1}, \ldots, \bar{Y}_{m}$ ( $\bar{X}$ is not included) and iterated Lie brackets of $X, \bar{Y}_{1}, \ldots, \bar{Y}_{m}$. Then, we can see that

$$
\bar{C}=\bar{C}_{0}+\operatorname{span}\{\bar{X}\}
$$

holds. We now prove (a) $\Rightarrow$ (i). We assume that there exist $n-k$ independent first integrals of the $A$-rheonomous affine constraints(2.3) on $Q: h_{1}(t, q), \ldots, h_{n-k}(t, q)$ and they satisfy (3.44)- 


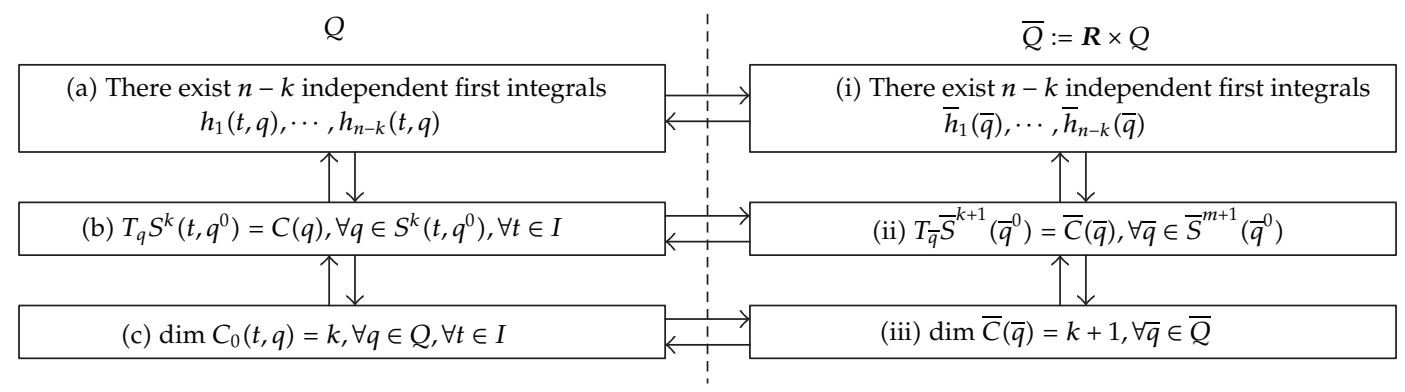

Figure 6: A diagram for the proof of Theorem 3.5.

(3.46). We only have to show that $n-k$ functions on $\bar{Q}: \bar{h}_{1}(\bar{q}):=h_{1}(t, q), \ldots, \bar{h}_{n-k}(\bar{q}):=$ $h_{n-k}(t, q)$ are independent first integrals of the expanded Pfaffian equation (3.17) on $\bar{Q}$, that is,

$$
\begin{gathered}
\bar{Z} \bar{h}_{i}=0, \quad \forall \bar{Z} \in \bar{C}, i=1, \ldots, n-k, \\
\overline{d h}_{1} \wedge \cdots \wedge \overline{d h}_{n-k} \neq 0
\end{gathered}
$$

hold. From (3.44), it turns out that $\bar{X} \bar{h}_{i}=0$ holds. In addition, we can see that $\bar{W} \in \bar{C}$ holds for $\forall \bar{W} \in \bar{C}_{0}$ from (3.45). Hence, by using (3.49), we obtain (3.50). By contrast, for (a) $\Leftarrow$ (i), since

$$
\begin{aligned}
\overline{d h}_{1} \wedge \cdots \wedge \overline{d h}_{n-k}= & \left(\frac{\partial h_{1}}{\partial t} d t+d h_{1}\right) \wedge \cdots \wedge\left(\frac{\partial h_{n-m}}{\partial t} d t+d h_{n-k}\right) \\
= & d h_{1} \wedge \cdots \wedge d h_{n-k}+\frac{\partial h_{1}}{\partial t} d t \wedge d h_{2} \wedge \cdots \wedge d h_{n-k} \\
& +\cdots+\frac{\partial h_{n-m}}{\partial t} d t \wedge d h_{1} \wedge \cdots \wedge d h_{n-k-1}
\end{aligned}
$$

holds, we have (3.51) under assumption (3.46). Therefore, it can be confirmed that $\bar{h}_{1}(\bar{q}), \ldots, \bar{h}_{n-k}(\bar{q})$ are $n-k$ independent first integrals of the expanded Pfaffian equation (3.18) on $\bar{Q}$. Consequently, we have proven (a) $\Leftrightarrow$ (i).

We next show the equivalence between (b) and (ii) and begin with the proof of (b) $\Leftarrow$ (ii). Denote by $\bar{S}^{k+1}\left(\bar{q}^{0}\right)$ a $(k+1)$-dimensional submanifold on $\bar{Q}$ with an initial point $\bar{q}^{0}$ and assume that

$$
T_{\bar{q}} \bar{S}^{k+1}\left(\bar{q}^{0}\right)=\bar{C}(\bar{q}), \quad \forall \bar{q} \in \bar{S}^{k+1}\left(\bar{q}^{0}\right)
$$


holds. By using the mapping $\pi_{t}$, which is introduced in the proof of Theorem 3.3, from (3.49) we obtain

$$
\begin{gathered}
d \pi_{t}\left(T_{\bar{q}} \bar{S}^{k+1}\left(\bar{q}^{0}\right)\right)=d \pi_{t}\left(T_{q} S^{k}(t, q)\right), \\
d \pi_{t}(\bar{C}(q))=d \pi_{t}\left(\bar{C}_{0}\right)=C_{0}(q),
\end{gathered}
$$

and hence from (3.53) we can show (3.47). Conversely, we can prove (b) $\Rightarrow$ (ii) by using the inverse mapping $\pi_{t}^{-1}$. So, the proof on (b) $\Leftrightarrow$ (ii) is completed.

Finally, we give the proof of the equivalence between (c) and (iii). We now prove (c) $\Rightarrow$ (iii). Since $\operatorname{dim} C_{0}=k>m$ holds from (3.48), we set

$$
C_{0}=\operatorname{span}\left\{Y_{1}, \ldots, Y_{m}, Y_{m+1}, \ldots, Y_{k}\right\}
$$

where $Y_{m+1}, \ldots, Y_{k}$ are $k-m$ independent vector fields on $Q$ that are independent of $Y_{1}, \ldots, Y_{m}$. Define vector fields on $\bar{Q}$ as $\bar{Y}_{i}:=0 \oplus Y_{i} \quad(i=m+1, \ldots, k)$. From (3.19) and (3.36), we can see that

$$
\bar{C}:=\operatorname{span}\left\{\bar{X}, \bar{Y}_{1}, \ldots, \bar{Y}_{m}, \bar{Y}_{m+1}, \ldots, \bar{Y}_{k}\right\}
$$

holds and hence we have $\operatorname{dim} \bar{C}=k+1$. This results in (c) $\Rightarrow$ (iii). For (c) $\Leftarrow$ (iii), the proof can be done by the inverse logic of the proof of (c) $\Rightarrow$ (iii). Hence, we have completed (c) $\Leftrightarrow$ (iii).

As a result, from the discussion above, we conclude that the statements (a)-(c) are equivalent to each other.

Note that similar to Theorem 3.3, conditions (a)-(c) in Theorem 3.5 are also analogous to the ones for the scleronomous affine constraints case [27, 29], and the role of the rheonomous affine bracket is quite important here. Moreover, condition (c) in Theorem 3.5 is also quite useful for checking the partial integrability of the given $A$-rheonomous affine constraints. In order to confirm Theorem 3.5, a simple example is now shown as follows.

Example 3.6. Consider a 3-dimensional configuration manifold

$$
Q=\left\{q=\left[\begin{array}{l}
q_{1} \\
q_{2} \\
q_{3}
\end{array}\right] \in \mathbf{R}^{3} \mid q_{2}>0, q_{3}>0\right\}
$$

with $n=3$ and $A$-rheonomous affine constraints on $Q$

$$
\left.\begin{array}{c}
t q_{1} q_{2} q_{3} \\
q_{2} q_{3}
\end{array}\right]+\left[\begin{array}{ccc}
q_{2} q_{3} & q_{1} q_{3} & q_{1} q_{2} \\
0 & 1 & q_{2}
\end{array}\right]\left[\begin{array}{l}
\dot{q}_{1} \\
\dot{q}_{2} \\
\dot{q}_{3}
\end{array}\right]=0
$$


with $m=1$. Moreover, we consider a time interval $I:=\mathbf{R}$. We can see that Assumption 2.1 holds for (3.58). One of the geometric representations of (3.58) can be obtained as follows:

$$
X(t, q)=\left[\begin{array}{c}
-t q_{1}+q_{1} q_{3} \\
-q_{2} q_{3} \\
0
\end{array}\right], \quad Y(q)=\left[\begin{array}{c}
-q_{1}+q_{1} q_{3} \\
-q_{2} q_{3} \\
q_{3}
\end{array}\right]
$$

By calculating the iterated rheonomous brackets of $X$ and $Y$ above, we have

$$
\begin{gathered}
\langle X, Y\rangle=\frac{\partial Y}{\partial t}+[X, Y]=0+\frac{\partial Y}{\partial q} X-\frac{\partial X}{\partial q} Y=\left[\begin{array}{c}
-q_{1} q_{3} \\
q_{2} q_{3} \\
0
\end{array}\right], \\
\langle X,\langle X, Y\rangle\rangle=\frac{\partial\langle X, Y\rangle}{\partial t}+[X,\langle X, Y\rangle]=\left[\begin{array}{c}
t q_{1} q_{3}-q_{1} q_{3}^{2} \\
-q_{2} q_{3}^{2} \\
0
\end{array}\right]-\left[\begin{array}{c}
t q_{1} q_{3}-q_{1} q_{3}^{2} \\
-q_{2} q_{3}^{2} \\
0
\end{array}\right]=0, \\
\langle Y,\langle X, Y\rangle\rangle=[Y,\langle X, Y\rangle]=\left[\begin{array}{c}
-q_{1} q_{3}^{2} \\
-q_{2} q_{3}^{2}+q_{2} q_{3} \\
0
\end{array}\right]-\left[\begin{array}{c}
q_{1} q_{3}-q_{1} q_{3}^{2} \\
-q_{2} q_{3}^{2} \\
0
\end{array}\right]=\left[\begin{array}{c}
-q_{1} q_{3} \\
q_{2} q_{3} \\
0
\end{array}\right],
\end{gathered}
$$

Therefore, we have

$$
C_{0}=\operatorname{span}\{Y,\langle X, Y\rangle\},
$$

and we can see that

$$
\operatorname{dim} C_{0}=2, \quad \forall q \in Q, \forall t \in I,
$$

holds. From Theorem 3.5, we can find that the $A$-rheonomous affine constraints (3.58) are 2nd order partial nonintegrable with $k=2$. In fact, there exists one first integral of (3.58):

$$
h_{1}(t, q)=\frac{q_{1} q_{2} q_{3} e^{(1 / 2) t^{2}}}{q_{2}^{0} q_{3}^{0}}-q_{1}^{0}
$$

where $q^{0}=\left[\begin{array}{lll}q_{1}^{0} & q_{2}^{0} & q_{3}^{0}\end{array}\right]^{\top} \in Q$ is an initial point at the initial time. We can easily confirm that (3.63) satisfies (3.12)-(3.14) of condition (a) in Theorem 3.5, and hence (3.63) are independent first integrals of (3.58). In the second paper [42], we will show how to derive (3.63) by using an integrating algorithm for partially integrable $A$-rheonomous affine constraints. 


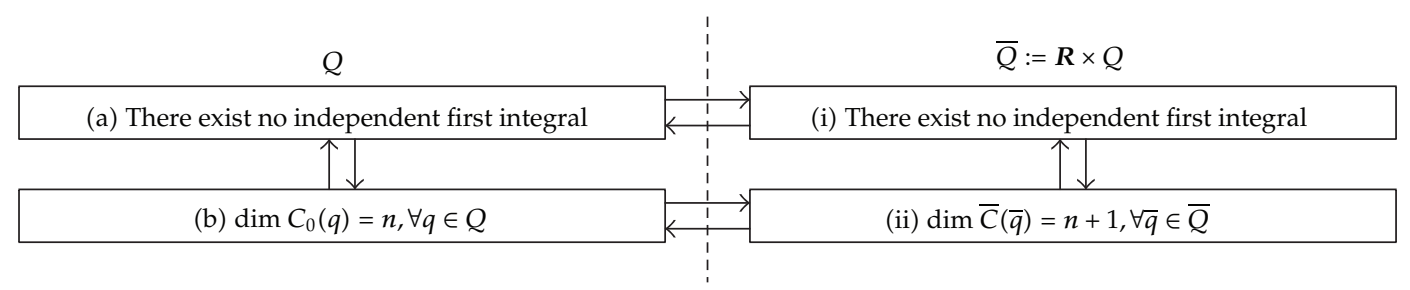

Figure 7: A diagram for the proof of Theorem 3.7.

\subsection{Complete Nonintegrability Conditions}

Finally, we deal with the remaining case where all the $A$-rheonomous affine constraints (2.3) are nonintegrable. This case is called complete nonintegrability. The complete nonintegrability case is quite important in nonholonomic control theory and sub-Riemannian geometry $[43,44]$. Some necessary and sufficient conditions on the complete nonintegrability of the $A$-rheonomous affine constraints (2.3) are shown in the next theorem.

Theorem 3.7. For the A-rheonomous affine constraints (2.3) defined on an n-dimensional manifold $Q$ and a time interval $I \subset \mathbf{R}$, the following statements $(a)$ and $(b)$ are equivalent to each other. If they hold, the A-rheonomous affine constraints (2.3) are said to be completely nonintegrable or completely nonholonomic.

(a) There do not exist independent first integrals of the A-rheonomous affine constraints (2.3).

(b) For a smallest and involutive time-varying distribution $C_{0}$,

$$
\operatorname{dim} C_{0}(t, q)=n, \quad \forall q \in Q, \forall t \in I,
$$

holds.

Proof. The diagram of the proof of Theorem 3.7 is shown in Figure 7. Similar to the proofs of Theorems 3.3 and 3.5, we can show that the statements (i) and (ii) in Figure 7 are equivalent to each other by using Frobenius' theorem [17, 19], and we only have to prove the two equivalences between (a) and (i), and (b) and (ii) in order to complete the proof of this theorem. First, we can find that (a) $\Leftrightarrow$ (i) obviously holds. Then, considering the case where $k=n$ in the proof of Theorem 3.5, we can also prove (b) $\Leftrightarrow$ (ii), and the details are omitted. Consequently, we conclude that the statements (a) and (b) are equivalent to each other.

From the results in Theorems 3.3-3.7, we can conclude that the integrability or nonintegrability of the given $A$-rheonomous affine constraints can be confirmed by calculating a smallest and involutive time-varying distribution $C_{0}$ with the rheonomous bracket and the conditions are similar to the ones for the scleronomous affine constraints case $[27,29]$ despite the explicit existence of the time variable in the $A$-rheonomous affine constraints. Now, we illustrate a simple example to check the availability of Theorem 3.7 as follows. 
Example 3.8. We here consider a 3-dimensional configuration manifold

$$
Q=\left\{q=\left[\begin{array}{l}
q_{1} \\
q_{2} \\
q_{3}
\end{array}\right] \in \mathbf{R}^{3}\right\}
$$

with $n=3$ and $A$-rheonomous affine constraints on $Q$

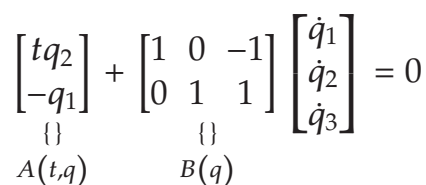

with $m=1$. Now, we consider a time interval $I:=(0, \infty]$. We can see that Assumption 2.1 holds for (3.66). One of the geometric representations of (3.66) can be obtained as follows:

$$
X(t, q)=\left[\begin{array}{c}
-t q_{2} \\
q_{1} \\
0
\end{array}\right], \quad Y(q)=\left[\begin{array}{c}
1 \\
-1 \\
1
\end{array}\right]
$$

Calculating the iterated rheonomous brackets of $X$ and $Y$ above, we have

$$
\begin{gathered}
\langle X, Y\rangle=\frac{\partial Y}{\partial t}+[X, Y]=0+\frac{\partial Y}{\partial q} X-\frac{\partial X}{\partial q} Y=\left[\begin{array}{c}
-t \\
0 \\
0
\end{array}\right] \\
\langle Y, X\rangle=-\frac{\partial Y}{\partial t}+[Y, X]=0+\left[\begin{array}{l}
t \\
0 \\
0
\end{array}\right] \\
\langle X,\langle X, Y\rangle\rangle=\frac{\partial\langle X, Y\rangle}{\partial t}+[X,\langle X, Y\rangle]=\left[\begin{array}{c}
-1 \\
0 \\
0
\end{array}\right]+\left[\begin{array}{l}
0 \\
t \\
0
\end{array}\right]=\left[\begin{array}{c}
-1 \\
t \\
0
\end{array}\right], \\
\langle\langle X, Y\rangle, X\rangle=-\frac{\partial\langle X, Y\rangle}{\partial t}+[\langle X, Y\rangle, X]=\left[\begin{array}{l}
1 \\
t \\
0
\end{array}\right] \\
\langle Y,\langle X, Y\rangle\rangle=[Y,\langle X, Y\rangle]=0 \\
\langle\langle X, Y\rangle, Y\rangle=[\langle X, Y\rangle, Y]=0
\end{gathered}
$$

Therefore, we have

$$
C_{0}=\operatorname{span}\{Y,\langle X, Y\rangle,\langle X,\langle X, Y\rangle\rangle\}
$$




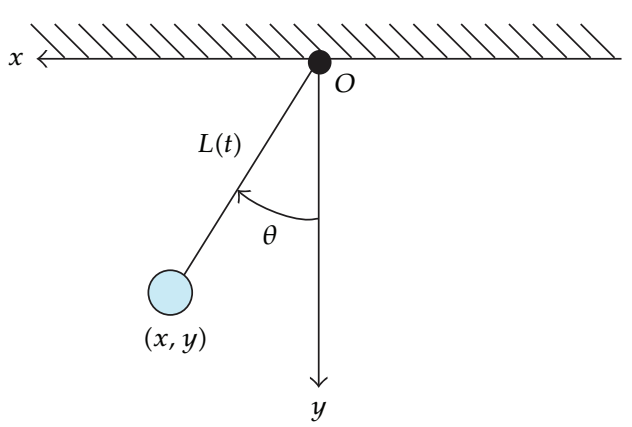

Figure 8: A pendulum with a time-varying elastic string.

and it can be confirmed that

$$
\operatorname{dim} C_{0}=3, \quad \forall q \in Q, \forall t \in I,
$$

holds. From Theorem 3.7, we can see that the A-rheonomous affine constraints (3.66) are completely nonintegrable, and hence there exist no independent first integrals of (3.66).

\section{Physical Examples}

\subsection{Pendulum with Time-Varying Elastic String}

In this section, we consider three types of physical examples in order to confirm the results obtained in the previous sections.

First, in this subsection, we deal with a pendulum with an elastic string as shown in Figure 8. This system is composed of a weight and an elastic string. One end of the string is fixed to the ceiling and can swing around the fixed point. The weight is installed at the other end of the string. As shown in Figure 8, set the $x$-axis and the $y$-axis to the horizontal and vertical directions, respectively. We also set the origin of the coordinate system so that it corresponds to the fixed point of the string. Therefore, we consider the 2-dimensional configuration manifold

$$
Q=\left\{q=\left[\begin{array}{l}
x \\
y
\end{array}\right] \in \mathbf{R}^{2} \mid y \geq 0\right\}
$$

with $n=2$. The position of the weight in $Q$ is represented by $(x, y)$, and the angle of the string in the clockwise direction measured from the $y$-axis is denoted by $\theta$. Now, we assume that the length of the string is changed as time goes by, that is, a time-varying elastic string, and we denote the length by $L(t)$.

Obviously, this system is subject to a constraint on the configuration variables as

$$
\sqrt{x^{2}+y^{2}}=L(t) .
$$


It is easily confirmed that since (4.2) does not contain any derivatives of the configuration variables, (4.2) is a rheonomous holonomic constraint. Hence, we can reduce the number of the configuration variables by replacing $(x, y)$ by $\theta$. However, we here deal with this system as a physical example that is subject to an integrable $A$-rheonomous affine constraint. Calculating time derivative of (4.2), we have

$$
x \dot{x}+y \dot{y}=\dot{L}(t) L(t)
$$

Now, for the sake of simplicity, we set $N(t):=\dot{L}(t) L(t)$. Then, (4.3) can be represented as an A-rheonomous affine constraint:

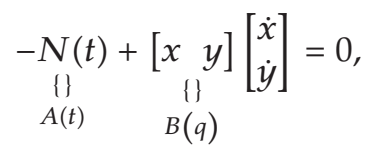

where $m=1$. We start with the above $A$-rheonomous affine constraint the (4.4). One of geometric representations of the $A$-rheonomous affine constraints (4.4) can be derived as

$$
X=\left[\begin{array}{c}
0 \\
\frac{N(t)}{y}
\end{array}\right], \quad Y=\left[\begin{array}{c}
1 \\
-\frac{x}{y}
\end{array}\right]
$$

For the vector fields $X$ and $Y$, we calculate a rheonomous bracket as

$$
\langle X, Y\rangle=\frac{\partial Y}{\partial t}+[X, Y]=\left[\begin{array}{c}
0 \\
\frac{N(t) x}{y^{3}}
\end{array}\right]-\left[\begin{array}{c}
0 \\
\frac{N(t) x}{y^{3}}
\end{array}\right]=\left[\begin{array}{l}
0 \\
0
\end{array}\right]
$$

and hence we can see that all the iterated rheonomous brackets of $X$ and $Y$ are 0 . Therefore, we have

$$
C_{0}=\operatorname{span}\{Y\}
$$

and so

$$
\operatorname{dim} C_{0}=1, \quad \forall q \in Q, \forall t \in I:=\mathbf{R},
$$

holds. From Theorem 3.3, it turns out that the A-rheonomous affine constraint (4.4) is completely integrable, and this result coincides with the problem setting of this system.

\subsection{Boat on Running River with Time-Varying Stream}

Next, we deal with another physical example called a boat on a running river as shown in Figure 9. Set the $x$-axis and the $y$-axis to the transverse direction and the downstream 


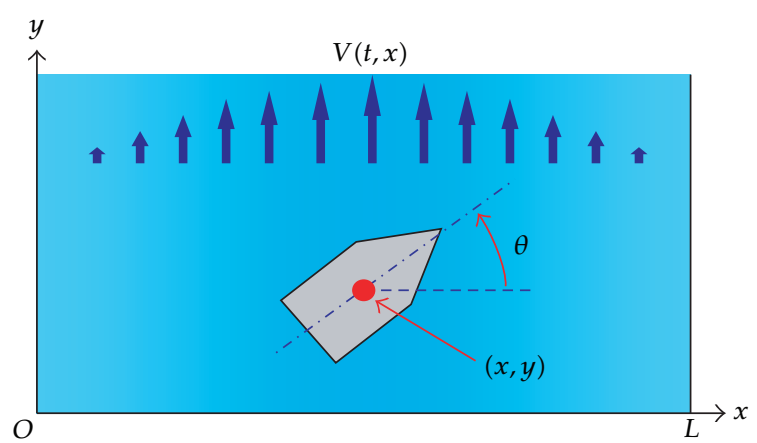

Figure 9: A boat on a running river with a time-varying stream.

direction of the river, respectively, and denote the center of inertia of the boat by $(x, y)$. In addition, let $\theta$ be the angle of the boat. Let $V(t, x)$ be a stream of the river that depends on the time variable $t$ as well as the transverse position $x$, that is, the stream changes as time goes by. It is assumed that the boat is affected by the stream to the downstream direction of the river according to the angle of the boat $\theta$, and hence the boat drifts to the $y$-direction. Then, the generalized coordinate of this system is represented by $q=\left[\begin{array}{lll}x & y & \theta\end{array}\right]^{\top} \in \mathbf{R}^{3}$, and we consider the 3-dimensional configuration manifold

$$
Q=\left\{q=\left[\begin{array}{l}
x \\
y \\
\theta
\end{array}\right] \in \mathbf{R}^{3} \mid 0 \leq x \leq L\right\}
$$

with $n=3$, where $L$ is the length of the river.

Considering the balance of the velocities in both the heading and side directions of the boat, we have the $A$-rheonomous affine constraints of this system as

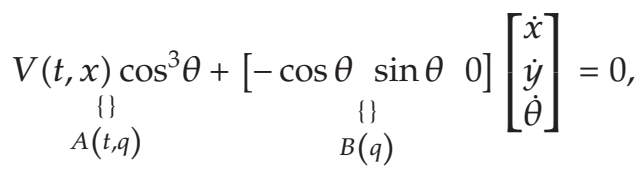

where $m=2$. We can derive one of the geometric representations of the $A$-rheonomous affine constraints (4.10) as

$$
X=\left[\begin{array}{c}
V(t, x) \cos ^{2} \theta \\
0 \\
0
\end{array}\right], \quad Y_{1}=\left[\begin{array}{c}
\sin \theta \\
\cos \theta \\
0
\end{array}\right], \quad Y_{2}=\left[\begin{array}{l}
0 \\
0 \\
1
\end{array}\right]
$$


For the vector fields $X, Y_{1}, Y_{2}$, calculate some iterated rheonomous brackets:

$$
\begin{aligned}
& \left\langle X, Y_{1}\right\rangle=\frac{\partial Y_{1}}{\partial t}+\left[X, Y_{1}\right]=\left[\begin{array}{c}
-\frac{\partial V}{\partial x} \sin \theta \cos ^{2} \theta \\
0 \\
0
\end{array}\right], \\
& \left\langle X, Y_{2}\right\rangle=\frac{\partial Y_{2}}{\partial t}+\left[X, Y_{2}\right]=\left[\begin{array}{c}
2 V(t, x) \sin \theta \cos \theta \\
0 \\
0
\end{array}\right], \\
& \left\langle Y_{1}, Y_{2}\right\rangle=\left[Y_{1}, Y_{2}\right]=\left[\begin{array}{c}
\cos \theta \\
-\sin \theta \\
0
\end{array}\right] .
\end{aligned}
$$

Therefore, we have

$$
C_{0}=\operatorname{span}\left\{Y_{1}, Y_{2},\left\langle X, Y_{1}\right\rangle,\left\langle X, Y_{2}\right\rangle,\left\langle Y_{1}, Y_{2}\right\rangle, \ldots\right\}
$$

and hence

$$
\operatorname{dim} C_{0}=3=n, \quad \forall q \in Q, \quad t \in I:=\mathbf{R},
$$

holds. Consequently, we conclude that the rheonomous affine constraints of this system (4.10) are completely nonholonomic from Theorem 3.7.

\subsection{Ball on Rotating Table at Time-Varying Angular Velocity}

Finally, let us deal with a ball on a rotating table in this subsection. We deal with an undeformable ball and a rotating table that turns at a time-varying angular velocity as shown in Figure 10. We assume that the ball does not slip and rotates with a velocity received by the rotating table. Consider the $x-y$ coordinate system so that its origin $O$ is coincident with the center of the rotating table, and let $(x, y)$ be the point with which the ball contacts. We denote the angles of rotation of the ball by using the Euler angles $(\theta, \phi, \psi)$. Hence, the generalized coordinate of this system is represented by $q=\left[\begin{array}{lllll}x & y & \theta & \phi & \psi\end{array}\right]^{\top} \in \mathbf{R}^{5}$, and we consider the 3-dimensional configuration manifold

$$
Q=\left\{q=\left[\begin{array}{l}
x \\
y \\
\theta \\
\phi \\
\psi
\end{array}\right] \in \mathbf{R}^{2} \times S O(3) \mid(x, y) \in \mathbf{R}^{2},(\theta, \phi, \psi) \in S O(3)\right\}
$$

with $n=5$.

We here assume that the angular velocity of the rotating table depends on the time variable and is denoted by $\Omega(t)$. In addition, we also denote the radius of the ball by $R$. 

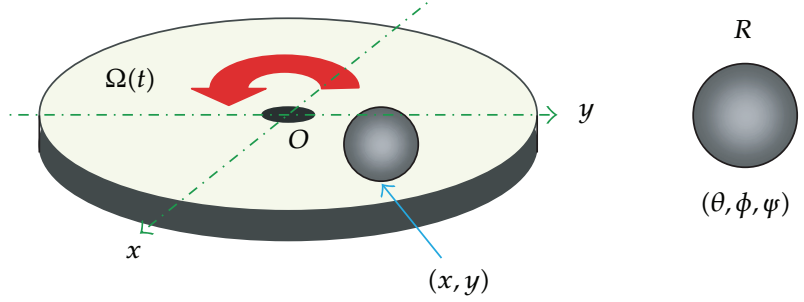

Figure 10: A ball on a rotating table at a time-varying angular velocity.

Considering the balance of the velocities in both $x$ and $y$ directions of the ball, we have the rheonomous affine constraints of this system as

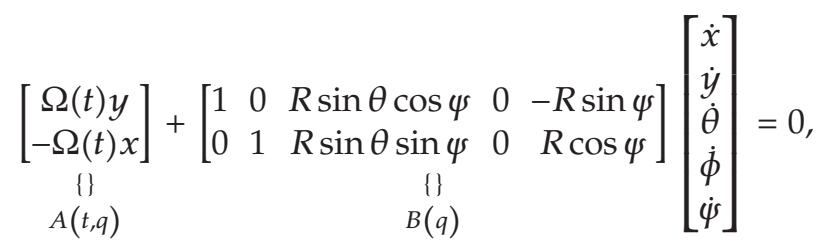

where $m=3$. We can derive one of the geometric representations of (4.16) as

$$
X=\left[\begin{array}{c}
\Omega(t) y \\
-\Omega(t) x \\
0 \\
0 \\
0
\end{array}\right], \quad Y_{1}=\left[\begin{array}{c}
-R \sin \theta \cos \psi \\
-R \sin \theta \sin \psi \\
1 \\
0 \\
0
\end{array}\right], \quad Y_{2}=\left[\begin{array}{l}
0 \\
0 \\
0 \\
1 \\
0
\end{array}\right], \quad Y_{3}=\left[\begin{array}{c}
R \sin \psi \\
-R \cos \psi \\
0 \\
0 \\
1
\end{array}\right]
$$

For the vector fields $X, Y_{1}, Y_{2}$, and $Y_{3}$ above, calculating some iterated rheonomous brackets, we can obtain

$$
\begin{gathered}
\left\langle X, Y_{1}\right\rangle=\frac{\partial Y_{1}}{\partial t}+\left[X, Y_{1}\right]=\left[\begin{array}{c}
R \Omega(t) \sin \theta \sin \psi \\
-R \Omega(t) \sin \theta \cos \psi \\
0 \\
0 \\
0
\end{array}\right], \\
\left\langle X, Y_{2}\right\rangle=\frac{\partial Y_{2}}{\partial t}+\left[X, Y_{2}\right]=0 \\
\left\langle X, Y_{3}\right\rangle=\frac{\partial Y_{3}}{\partial t}+\left[X, Y_{3}\right]=\left[\begin{array}{c}
R \Omega(t) \cos \psi \\
R \Omega(t) \sin \psi \\
0 \\
0 \\
0
\end{array}\right], \\
\left\langle Y_{1}, Y_{2}\right\rangle=\left[Y_{1}, Y_{2}\right]=0, \quad\left\langle Y_{2}, Y_{3}\right\rangle=\left[Y_{2}, Y_{3}\right]=0,
\end{gathered}
$$




$$
\begin{array}{r}
\left\langle Y_{1}, Y_{3}\right\rangle=\left[Y_{1}, Y_{3}\right]=\left[\begin{array}{c}
-R \sin \theta \sin \psi \\
R \sin \theta \cos \psi \\
0 \\
0 \\
0
\end{array}\right], \\
\left\langle Y_{1},\left\langle Y_{1}, Y_{3}\right\rangle\right\rangle=\left[Y_{1},\left[Y_{1}, Y_{3}\right]\right]=\left[\begin{array}{c}
-R \cos \theta \sin \psi \\
R \cos \theta \cos \psi \\
0 \\
0 \\
0
\end{array}\right], \\
\left\langle Y_{3},\left\langle Y_{1}, Y_{3}\right\rangle\right\rangle=\left[Y_{3},\left[Y_{1}, Y_{3}\right]\right]=\left[\begin{array}{c}
-R \sin \theta \cos \psi \\
-R \sin \theta \sin \psi \\
0 \\
0 \\
0
\end{array}\right] .
\end{array}
$$

Consequently, we can see that

$$
C_{0}=\operatorname{span}\left\{Y_{1}, Y_{2}, Y_{3},\left\langle Y_{1}, Y_{3}\right\rangle,\left\langle Y_{1},\left\langle Y_{1}, Y_{3}\right\rangle\right\rangle,\left\langle Y_{3},\left\langle Y_{1}, Y_{3}\right\rangle\right\rangle, \ldots\right\}
$$

holds and hence

$$
\operatorname{dim} C_{0}=5=n, \quad \forall q \in Q, \forall t \in I:=\mathbf{R},
$$

holds. Consequently, from Theorem 3.7, it turns out that the rheonomous affine constraints of this system (4.16) are completely nonintegrable.

\section{Conclusions}

In this paper, we have dealt with the $A$-rheonomous affine constraints from the standpoint of integrability/nonintegrability. First, some fundamental properties of the $A$-rheonomous affine constraints have been obtained. We next have investigated the three cases, complete integrability, partial integrability, and complete nonintegrability for the $A$-rheonomous affine constraints and derived some necessary and sufficient conditions for the respective cases by using the rheonomous bracket that is a newly proposed operator. Some physical examples show the effectiveness and the application potentiality of our new results.

From these results, we have mainly confirmed the three important results as follows: (a) though the $A$-rheonomous affine constraints contain the time variable explicitly, the necessary and sufficient conditions on their integrability/nonintegrability are similar to the ones for the scleronomous affine constraints [27], (b) we can easily determine the integrability/nonintegrability of the given $A$-rheonomous affine constraints by calculating a time-varying distribution $C_{0}$ and (c) the rheonomous bracket plays important roles in the 
conditions. We can say that the results obtained in this paper present useful tools for not only control theory but also various research fields such as general mathematics and physics.

In our second paper [42], we will investigate the relationship between the integrability/nonintegrability of the $A$-rheonomous affine constraints and foliation structures of configuration manifolds. Moreover, we will develop integrating algorithms that are used to calculate independent first integrals for integrable $A$-rheonomous affine constraints.

\section{Acknowledgment}

This work was partially supported by Kurata Grants.

\section{References}

[1] I. Ju. Neimark and N. A. Fafaev, Dynamics of Nonholonomic Systems, American Mathematical Society, 1972.

[2] Z. Li and J. F. Canny, Nonholonomic Motion Planning, Kluwer Academic Publishers, 1993.

[3] J. Cortés Monforte, Geometric, Control and Numerical Aspects of Nonholonomic Systems, vol. 1793 of Lecture Notes in Mathematics, Springer, Berlin, Germany, 2002.

[4] A. M. Bloch, Nonholonomic Mechanics and Control, vol. 24 of Interdisciplinary Applied Mathematics, Springer, New York, NY, USA, 2003.

[5] F. Bullo and A. D. Rewis, Geometric Control of Mechanical Systems, Springer Science, Business Media, 2004.

[6] C. Canudas de Wit and O. J. Sørdalen, "Exponential stabilization of mobile robots with nonholonomic constraints," Institute of Electrical and Electronics Engineers, vol. 37, no. 11, pp. 1791-1797, 1992.

[7] R. M. Murray and S. S. Sastry, "Nonholonomic motion planning: steering using sinusoids," Institute of Electrical and Electronics Engineers, vol. 38, no. 5, pp. 700-716, 1993.

[8] D. Tilbury, R. M. Murray, and S. S. Sastry, "Trajectory generation for the N-trailer problem using Goursat normal form," Institute of Electrical and Electronics Engineers, vol. 40, no. 5, pp. 802-819, 1995.

[9] C. Altafini, A. Speranzon, and B. Wahlberg, "A feedback control scheme for reversing a truck and trailer vehicle," IEEE Transactions on Robotics and Automation, vol. 17, no. 6, pp. 915-922, 2001.

[10] C. Fernandes, L. Gurvits, and Z. X. Li, "Near-optimal nonholonomic motion planning for a system of coupled rigid bodies," Institute of Electrical and Electronics Engineers, vol. 39, no. 3, pp. 450-463, 1994.

[11] X. S. Ge and L. Q. Chen, "Attitude control of a rigid spacecraft with two momentum wheel actuators using genetic algorithm," Acta Astronautica, vol. 55, no. 1, pp. 3-8, 2004.

[12] J.-M. Godhavn, A. Balluchi, L. S. Crawford, and S. S. Sastry, "Steering of a class of nonholonomic systems with drift terms," Automatica, vol. 35, no. 5, pp. 837-847, 1999.

[13] T. Mita, S. H. Hyon, and T. K. Nam, "Analytical time optimal control solution for a two-link planar acrobot with initial angular momentum," IEEE Transactions on Robotics and Automation, vol. 17, no. 3, pp. 361-366, 2001.

[14] M. D. Berkemeier and R. S. Fearing, "Sliding and hopping gaits for the underactuated acrobot," IEEE Transactions on Robotics and Automation, vol. 14, no. 4, pp. 629-634, 1998.

[15] M. D. Berkemeier and R. S. Fearing, "Tracking fast inverted trajectories of the underactuated Acrobot," IEEE Transactions on Robotics and Automation, vol. 15, no. 4, pp. 740-750, 1999.

[16] M. Reyhanoglu, A. van der Schaft, N. H. McClamroch, and I. Kolmanovsky, "Dynamics and control of a class of underactuated mechanical systems," Institute of Electrical and Electronics Engineers, vol. 44, no. 9, pp. 1663-1671, 1999.

[17] A. Isidori, Nonlinear Control Systems, Communications and Control Engineering Series, Springer, Berlin, Germany, 3rd edition, 1995.

[18] S. S. Sastry, Nonlinear Systems, vol. 10, Springer, New York, NY, USA, 1999.

[19] H. Nijmeijer and A. van der Schaft, Nonlinear Dynamical Control Systems, Springer, New York, NY, USA, 1990.

[20] V. Jurdjevic, Geometric Control Theory, vol. 52 of Cambridge Studies in Advanced Mathematics, Cambridge University Press, Cambridge, UK, 1996. 
[21] A. M. Bloch, M. Reyhanoglu, and N. H. McClamroch, "Control and stabilization of nonholonomic dynamic systems," Institute of Electrical and Electronics Engineers, vol. 37, no. 11, pp. 1746-1757, 1992.

[22] R. M. Murray, "Nilpotent bases for a class of nonintegrable distributions with applications to trajectory generation for nonholonomic systems," Mathematics of Control, Signals, and Systems, vol. 7, no. 1, pp. 58-75, 1994.

[23] O. J. Sørdalen and O. Egeland, "Exponential stabilization of nonholonomic chained systems," Institute of Electrical and Electronics Engineers, vol. 40, no. 1, pp. 35-49, 1995.

[24] A. J. van der Schaft and B. M. Maschke, "On the Hamiltonian formulation of nonholonomic mechanical systems," Reports on Mathematical Physics, vol. 34, no. 2, pp. 225-233, 1994.

[25] K. Fujimoto and T. Sugie, "Stabilization of Hamiltonian systems with nonholonomic constraints based on time-varying generalized canonical transformations," Systems E Control Letters, vol. 44, no. 4, pp. 309-319, 2001.

[26] R. W. Brockett, "Asymptotic stability and feedback stabilization," in Differential Geometric Control Theory, vol. 27 of Progress in Mathematics, pp. 181-191, Birkhäuser, Boston, Mass, USA, 1983.

[27] T. Kai and H. Kimura, "Theoretical analysis of affine constraints on a configuration manifold—part I : integrability and nonintegrability conditions for affine constraints and foliation structures of a configuration manifold," Transactions of the Society of Instrument and Control Engineers, vol. 42, no. 3, pp. 212-221, 2006.

[28] T. Kai, "Integrating algorithms for integrable affine constraints," IEICE Transactions on Fundamentals of Electronics, Communications and Computer Sciences, vol. E94-A, no. 1, pp. 464-467, 2011.

[29] T. Kai and H. Kimura, "Theoretical analysis of affine constraints on a configuration manifold-part II : accessibility of kinematic asymmetric affine control systems with affine constraints," Transactions of the Society of Instrument and Control Engineers, vol. 42, no. 3, pp. 222-231, 2006.

[30] T. Kai, H. Kimura, and S. Hara, "Nonlinear control analysis on kinematically asymmetrically affine control systems with nonholonomic affine constraints," in Proceedings of the 16th Triennial World Congress of International Federation of Automatic Control (IFAC '05), pp. 157-162, Czech Republic, July 2005.

[31] T. Kai, "Affine constraints in nonlinear control theory," in Proceedings of the 3rd Workshop on Lagrangian and Hamiltonian Methods for Nonlinear Control, pp. 251-256, Nagoya, Japan, 2006.

[32] T. Kai, "Extended chained forms and their application to nonholonomic kinematic systems with affine constraints: control of a coin on a rotating table," in Proceedings 45th IEEE Conference on Decision and Control (CDC '06), pp. 6104-6109, usa, December 2006.

[33] T. Kai, H. Kimura, and S. Hara, "Nonlinear control analysis on nonholonomic dynamic systems with affine constraints," in Proceedings of the 44th IEEE Conference on Decision and Control, and the European Control Conference (CDC-ECC '05), pp. 1459-1464, December 2005.

[34] T. Kai, "Derivation and analysis of nonholonomic hamiltonian systems with affine constraints," in Proceedings of the European Control Conference, pp. 4805-4810, Kos, Greece, 2007.

[35] T. Kai, "Generalized canonical transformations and passivity-based control for nonholonomic Hamiltonian systems with affine constraints: control of a coin on a rotating table," in Proceedings of the 46th IEEE Conference on Decision and Control(CDC '07), pp. 3369-3374, December 2007.

[36] T. Kai and K. Tamaki, "A near-optimal control approach to 3D ball-in-socket joint space robot models with initial angular momenta," Acta Astronautica, vol. 68, no. 11-12, pp. 1702-1711, 2011.

[37] W. Sarlet, F. Cantrijn, and D. J. Saunders, "A geometrical framework for the study of non-holonomic Lagrangian systems," Journal of Physics A, vol. 28, no. 11, Article ID 3253, 1995.

[38] D. J. Saunders, W. Sarlet, and F. Cantrijn, "A geometrical framework for the study of non-holonomic Lagrangian systems. II," Journal of Physics A, vol. 29, no. 14, pp. 4265-4274, 1996.

[39] M. de León, J. C. Marrero, and D. Martín de Diego, "Non-holonomic Lagrangian systems in jet manifolds," Journal of Physics A, vol. 30, no. 4, pp. 1167-1190, 1997.

[40] G. Giachetta, L. Mangiarotti, and G. Sardanashvily, "Nonholonomic constraints in time-dependent mechanics," Journal of Mathematical Physics, vol. 40, no. 3, pp. 1376-1390, 1999.

[41] F. Cantrijn, M. de León, J. C. Marrero, and D. Martín de Diego, "On almost-Poisson structures in nonholonomic mechanics. II. The time-dependent framework," Nonlinearity, vol. 13, no. 4, pp. 1379 1409, 2000.

[42] T. Kai, "Theoretical analysis for a class of rheonomous affine constraints on configuration manifolds —part II : foliation structures and integrating algorithms," Mathematical Problems in Engineering, vol. 2012, Article ID 345942, 2012.

[43] R. Montgomery, A Tour of Subriemannian Geometries, Their Geodesics and Applications, vol. 91 of Mathematical Surveys and Monographs, American Mathematical Society, Providence, RI, USA, 2002. 
[44] O. Calin and D.-C. Chang, Sub-Riemannian Geometry: General Theory and Examples, vol. 126 of Encyclopedia of Mathematics and Its Applications, Cambridge University Press, Cambridge, 2009. 


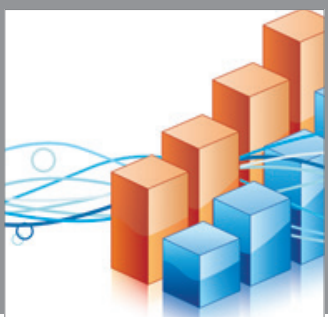

Advances in

Operations Research

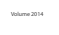

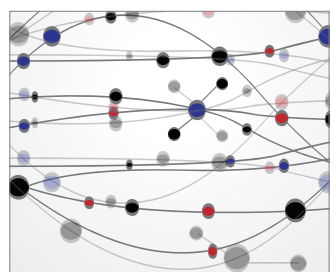

\section{The Scientific} World Journal
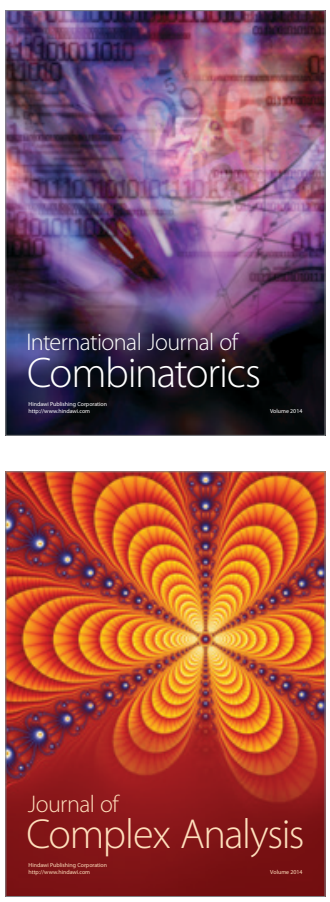

International Journal of

Mathematics and

Mathematical

Sciences
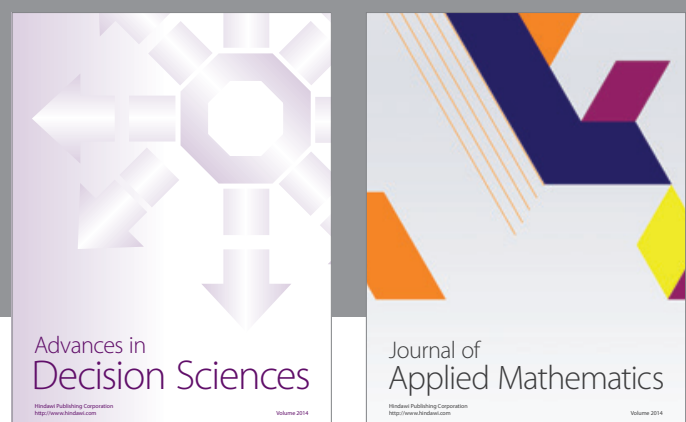

Journal of

Applied Mathematics
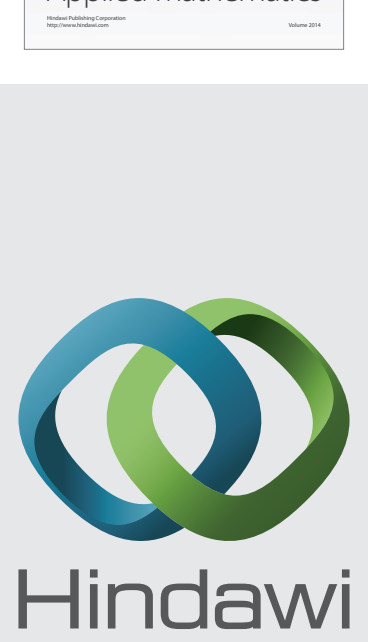

Submit your manuscripts at http://www.hindawi.com
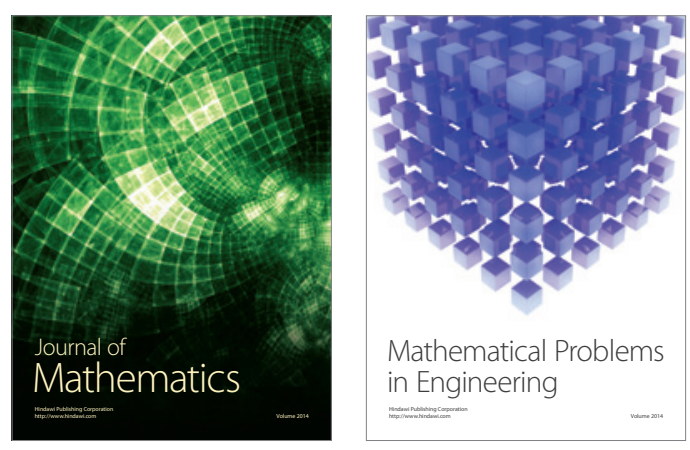

Mathematical Problems in Engineering
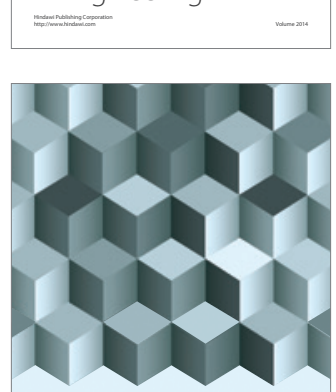

Journal of

Function Spaces
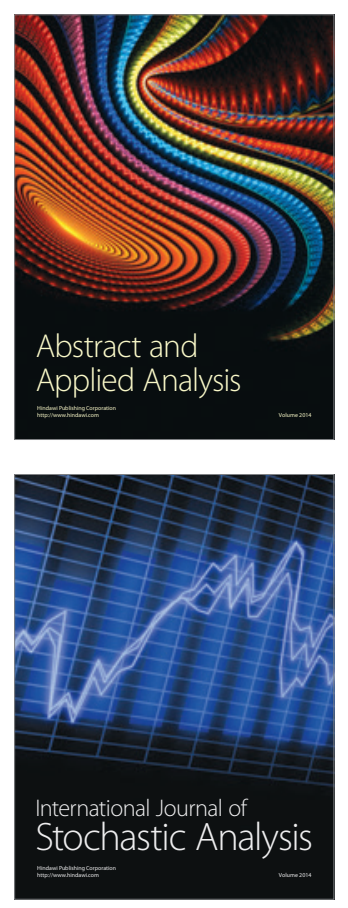

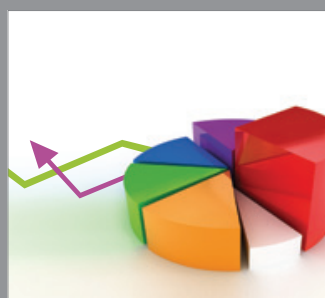

ournal of

Probability and Statistics

Promensencen
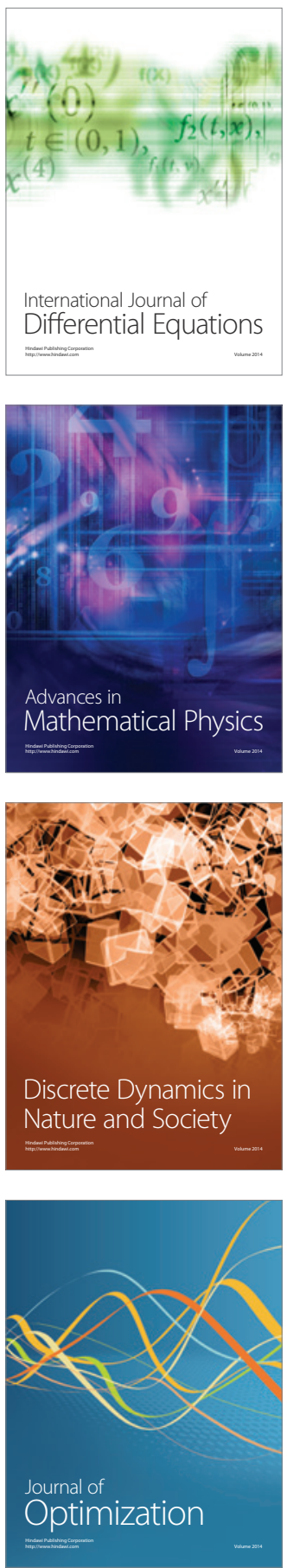